Viewpoint

\title{
Subdivided Cellular Automata Model Considering Anticipation Floor Field and Analysis of Pedestrian Detour Behavior
}

\author{
Jinrui Liu* $*$, Maosheng Li and Panpan Shu
}

check for

updates

Citation: Liu, J.; Li, M.; Shu, P. Subdivided Cellular Automata Model Considering Anticipation Floor Field and Analysis of Pedestrian Detour Behavior. Sustainability 2021, 13, 10621. https://doi.org/10.3390/ su131910621

Received: 27 August 2021

Accepted: 22 September 2021

Published: 24 September 2021

Publisher's Note: MDPI stays neutral with regard to jurisdictional claims in published maps and institutional affiliations.

Copyright: (c) 2021 by the authors. Licensee MDPI, Basel, Switzerland. This article is an open access article distributed under the terms and conditions of the Creative Commons Attribution (CC BY) license (https:/ / creativecommons.org/licenses/by/ $4.0 /)$.
School of Traffic and Transportation Engineering, Central South University, Changsha 410083, China; maosheng.li@csu.edu.cn (M.L.); panpanshu1@126.com (P.S.)

* Correspondence: a8214505a@163.com; Tel.: +86-189-7344-5926

\begin{abstract}
The micro-pedestrian simulation model represented by the cellular automata model is an important simulation model. Improvements in various aspects enable a better description of the various behaviors of pedestrians, such as pedestrian avoidance behavior, companion behavior, as well as transcendence behavior, waiting behavior and detour behavior. This paper takes the pedestrian detour behavior in the circle antipode experiment as the main entry point. The subdivision cellular automaton model is integrated into the prediction field to model and simulate the detour behavior. At the same time, it explores the degree of subdivision of the cell. Pedestrian heterogeneity and the influence of predicted field potential energy on the simulated pedestrian trajectory. Finally, based on the temporal and spatial indicators of pedestrian trajectory characteristics, the KS test and DTW method are used to evaluate the similarity of the trajectory distribution characteristics and time series characteristics with experimental samples, and evaluate and compare models with or without heterogeneity. The results show that the trajectory characteristics of heterogeneous pedestrians are closer to the experiment than homogeneous pedestrians.
\end{abstract}

Keywords: subdivided cells; heterogeneity; evaluation of trajectory characteristics

\section{Introduction}

The cellular automata model is a grid dynamic model in which time, space, and state are discrete, and the spatial interaction and time causality are local. It has the ability to simulate the spatiotemporal evolution process of complex systems. The cellular automata model is a classic discrete model. The model divides the scene into discrete cells. A single pedestrian occupies a single cell, and the pedestrian walks to its neighbor cells according to a specific rule or probability. Cellular automata models mainly include lattice gas model [1] and field potential energy model [2]. The cellular automata model is widely used in pedestrian simulation because of its flexibility, and has become one of the important models for studying pedestrian behavior.

In order to describe the specific behavior of pedestrians, many scholars have improved the cellular automata model, for example by introducing the role of social forces [3], subdividing the cell size [4] to finely describe the location of pedestrians and obstacles, and considering the heterogeneity of pedestrians [5].

The role of introducing social forces is mainly used to describe pedestrian behavior in evacuation scenes. Pedestrians will show different behaviors from general scenes in evacuation scenes such as exit scenes, which are mainly manifested in more intense conflicts with other pedestrians. Guo and Huang [6] combined the advantages of the lattice gas model with the social force model. The grid and neighbors of pedestrians are determined by the position of the pedestrians. The mutual repulsion between pedestrians is similar to that in the social force model. The evacuation scene simulation of a single exit is used. It is shown that this model can more accurately describe the movement characteristics of 
pedestrians at the exit, and can obtain a more accurate evacuation time. Chen et al. [7] concluded that, by simulating a classroom with two exits, the avoidance behavior has a negative impact on the efficiency of evacuation. The influence of the number of walkways in the classroom on the evacuation time is also studied, which is the layout of the building. Provided for reference. Other improvements are mainly used to describe other behaviors of pedestrians in general scenes. Zhou et al. [8] proposed a visual equation of pedestrians, and used the synchronized updated cellular automaton model to analyze pedestrians with speed heterogeneity. To describe the transcending behavior in the aisle, Davidich et al. [9] used a hexagonal cell form to set a waiting cell area in the subway station, and described the waiting behavior of pedestrians waiting for the subway to arrive. Fu et al. [10] described the turning behavior and lateral movement behavior of pedestrians at the bottleneck through the subdivided field potential cellular automata model, in order to better solve the deadlock phenomenon of pedestrians at the bottleneck. Zhuang et al. [11] described the pedestrian self-organization queuing behavior at the bottleneck without human services.

The speed heterogeneity of pedestrians is that pedestrians have different expected speeds due to factors such as age and height in the process of movement. In the traditional cellular automata model, pedestrians move one cell at each step, and the speed of pedestrians is the same, which is the same as that of actual pedestrians. The speed is inconsistent. Because pedestrian speed is related to step frequency and step length, there are two main ways to achieve the heterogeneity of pedestrian speed: the first is to change the step length of the pedestrian, that is, the distance that the pedestrian moves in the same time is different; the second is in order to change the pedestrian's cadence, that is, the time it takes for the pedestrian to move the same distance is different.

The first method mainly sets the maximum moving cell number $n$ per time step $s$ according to the pedestrian's expected speed, and the cell size is $k * k$. The pedestrian obtained by this method has $\mathrm{n}$ different speeds. of which the largest speed is $n * k / s . n$ represents the maximum number of moving cells, $k$ represents the size of the cell, and $s$ is the time step for pedestrians to move once. Guo [12] proposes the cellular automata model based on a subdivision of models which measures different expected speeds of pedestrians by changing the maximum number of pedestrian movement cells, and explores the simulation effects of subdivision and pedestrian speed on single exits. This method can directly, simply, and effectively present the speed heterogeneity of pedestrians, but its speed options are limited. The second method is to change the timescale of the time step of the pedestrian moving a cell, and let the pedestrian make a decision to walk or not to walk at each step through the movement probability so that the pedestrian can update his position completely at random to achieve his own speed. Compared with the first method, the speed heterogeneity of pedestrians can achieve more realistic distribution characteristics, and the calculation method is simple and the calculation burden is small. Zhou et al. proposed a synchronously updated cellular automaton model. Pedestrians change their step frequency according to their speed to realize the speed heterogeneity of pedestrians. In addition, visual equations are added and a series of rules are used to determine the beyond the behavior described, we explore the impact of pedestrian flow and pedestrian density of visual simulation speed of different groups of people. Ref [13] described the pedestrian heterogeneity in detail based on the subdivided field potential energy cellular automata model, including pedestrian speed heterogeneity, anisotropy, and time-varying characteristics such as fatigue. Among them, whether the pedestrian moves at each step and whether the turn is determined by the movement equation. The position of the pedestrian is updated completely randomly according to its expected speed, so as to realize the speed heterogeneity of the pedestrian.

In addition to pedestrian speed heterogeneity, there are other characteristics of pedestrian heterogeneity, such as baggage preference, proposed by Li et al. [14]; prediction range heterogeneity, suggested by Suma et al.; and left-right avoidance preference, put forward by Guo et al. [15]. It also has a greater impact on the movement characteristics of pedestrians. In this study, we consider the pedestrian speed and the heterogeneity of the 
prediction range, and propose a subdivision field potential energy cellular automata model integrated into the prediction field, which effectively reflects the pedestrian's prediction and avoidance behavior of the congested area, and better reproduces the pedestrian's detour behavior in the circular symmetry walking experiment. At the same time, for the temporal and spatial characteristics of pedestrian trajectories in the process of motion, we evaluated the similarity of the distribution and time series characteristics based on the Kolmogorov-Smirnov (KS) test and the dynamic time warping (DTW) method, and obtained the comparison of the advantages and disadvantages of the model simulation.

In the second section, the basic settings of the model are described in detail, an improved potential energy of the prediction field is proposed, and the heterogeneity of the pedestrian prediction range is realized through the subdivided cells. Then, in the third section, the scene setting and simulation of the circular symmetrical walking experiment are carried out to explore the influence of the degree of subdivision and the predicted field potential energy on the simulation. The fourth section evaluates the simulation effect based on the simulation trajectory data, the distribution characteristics, and the time series characteristics, and obtains the score comparison of the heterogeneous subdivision cellular automata model. In the last fifth section, the corresponding conclusions are drawn.

\section{Model Description}

\subsection{Cellular Automata Model of Field Potential Energy}

Subdividing a single cell can more accurately describe the location of pedestrians. The degree of subdivision is the degree to which the traditional cell is subdivided, set to $\mathrm{n}$. According to the size of the pedestrian, if the pedestrian occupies a single cell, the size of the traditional cell is generally $0.4 \times 0.4\left(\mathrm{~m}^{\wedge} 2\right)$. In setting the degree of subdivision, if it is $\mathrm{n}$, pedestrians occupy $\mathrm{n} \times \mathrm{n}$ cells, and the size of the subdivided cell is $0.4 / \mathrm{n} \times 0.4 / \mathrm{n}\left(\mathrm{m}^{\wedge} 2\right)$. Figure 1 shows a schematic diagram of pedestrian cells when the degree of subdivision is $\mathrm{n}=1$.

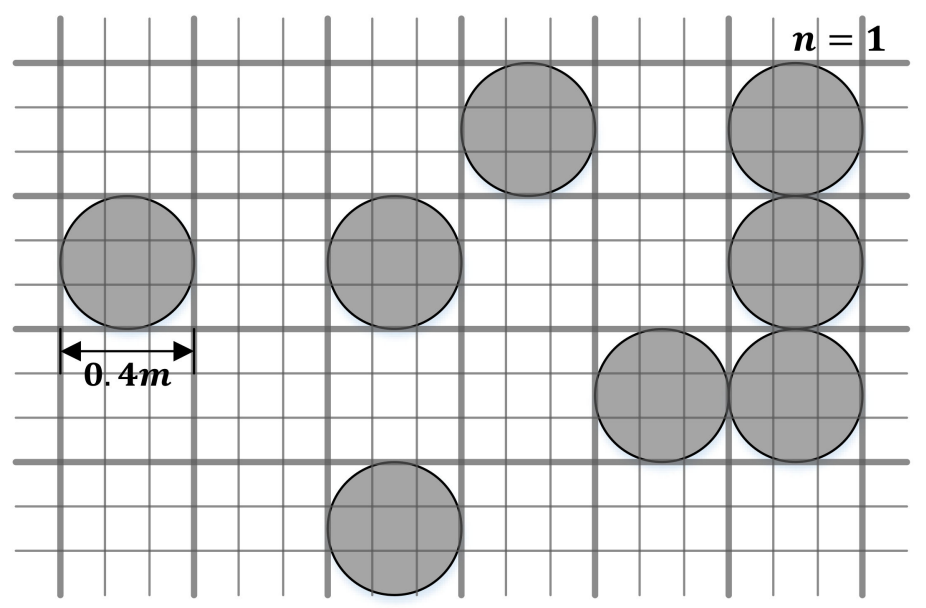

Figure 1. Traditional cell display of pedestrian distribution.

The subdivided cells can describe the layout of buildings and the location of pedestrians more precisely, and the distribution of pedestrians is more reasonable in the case of pedestrian congestion, as shown in Figure 2.

Comparing Figures 1 and 2, it can be seen that the subdivided cells can describe more precise pedestrian positions, the distribution of pedestrians is more reasonable, and, for scenes with higher local density, the fine description of pedestrian positions has a significant impact on the experimental results. 


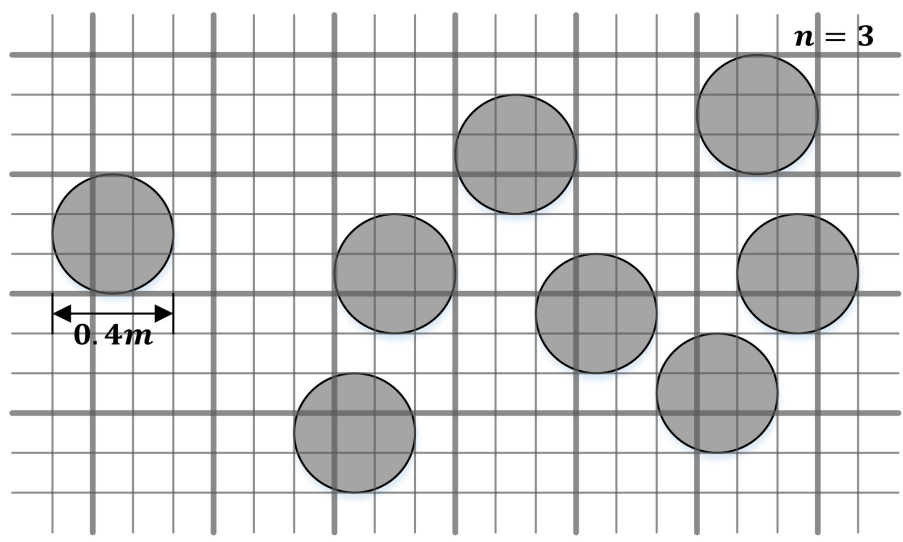

Figure 2. Pedestrian distribution subdivision cell display diagram.

The field potential energy transforms a large-scale interaction into a short-distance local effect. The motion of a pedestrian is determined by the static field potential energy $S_{i j}$ (its value does not change with time) and the dynamic field potential energy $D_{i j}$ (its value will change with time).

The static field potential energy (SFF) represents the attraction of the target point (exit) to pedestrians. It is calculated by the distance between the cell and the exit (target point) cell. The closer to the exit, the larger the SFF value $S_{i j}$ of the cell $(i, j)$. The calculation method is shown in Equation (1).

$$
S_{i, j}=\min _{\left(i_{\tau_{S}}, j_{\tau_{S}}\right)}\left\{\max _{\left(i_{1}, j_{1}\right)}\left\{\sqrt{\left(i_{\tau_{S}}-i_{1}\right)^{2}+\left(j_{\tau_{S}}-j_{1}\right)^{2}}\right\}-\sqrt{\left(i_{\tau_{S}}-i\right)^{2}+\left(j_{\tau_{S}}-j\right)^{2}}\right\}
$$

In the formula, $\left(i_{l}, j_{l}\right)$ is to traverse all the cells to find the cell with the maximum distance from the exit (target) cell $\left(i_{\tau_{s}}, j_{\tau_{s}}\right)$ ).

Dynamic field potential energy (DFF) can be regarded as the virtual trajectory left by pedestrians when they move, which expands to their neighbor cells and dissipates over time. DFF represents the behavioral characteristics of pedestrians following the path of others, that is, following behavior. When the pedestrian leaves the current cell, the DFF value $D_{i j}$ of the current cell is increased by one, that is, $D_{i j}(t+1)=D_{i j}(t)+1$. At the same time, its DFF value $\left(D_{i j}\right)$ expands and dissipates with a certain probability along with the time step, the expansion probability is $\delta$, and the dissipation probability is $\alpha$. That is, $D_{i j}(t+1)-D_{i j}(t)=\alpha \cdot \Delta D_{i j}(t)-\delta \cdot D_{i j}(t)$.

\subsection{Pedestrian Speed Heterogeneity}

Step length and step frequency are the main factors related to the speed heterogeneity of pedestrians. There are two main ways to realize the speed heterogeneity of pedestrians. The first is to change the step length of pedestrians, that is, pedestrians can walk more than one cell per step. The second is to change the pace of the pedestrian, that is, the faster the pedestrian, the less time it takes to walk the same distance.

A subdivided cellular automaton model is proposed. In order to realize the speed heterogeneity of pedestrians, a parameter $n$ is proposed to describe the degree of pedestrian subdivision, and a parameter $\mathrm{s}$ is proposed to describe the maximum movement step length of a pedestrian. As shown in Figure 3, under the subdivision $\mathrm{n}=3$, the size of each cell is $0.4 / 3 \times 0.4 / 3\left(\mathrm{~m}^{2}\right)$, and the pedestrian $P_{i}$ occupies $3 \times 3$ cells, due to the maximum moving step size $\mathrm{s}=4$, pedestrian $P_{i}$ has four different speed options. The figure shows two different speed options for pedestrians, $s_{i}=2$ (blue circle in the figure) and $s_{i}=4$ (red circle in Figure 3). 


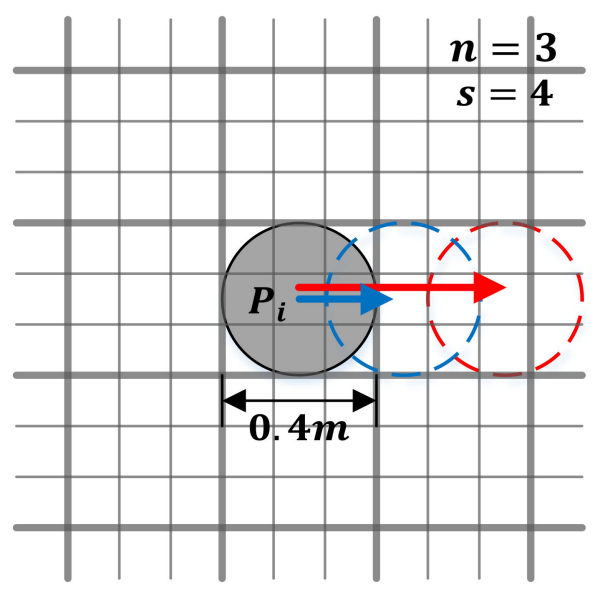

Figure 3. Schematic diagram of determining the speed heterogeneity of pedestrians with the maximum step size of the subdivided cells.

Although the above method of determining the speed heterogeneity of pedestrians by the maximum moving step length is simple to calculate, it only has a limited number of speed options and cannot describe the heterogeneity of human speed well. The size of the time step is changed according to the maximum walking speed, so as to ensure that the same distance is walked in a shorter time, and each pedestrian decides to walk or not to walk at each step according to the speed ratio. Pedestrians with high expected speed are easier to move. The movement of pedestrians is completely random. The size of the time step is determined by Equation (2):

$$
\Delta t=a / v_{\max }
$$

In the formula, a is the size of the cell, which is $0.4 / \mathrm{n} . v_{\max }$ is the maximum travel speed, which is set to $2(\mathrm{~m} / \mathrm{s})$ in this study.

In this article, we explore the impact of pedestrian speed heterogeneity on the simulation. We set the pedestrian's expected speed $v_{\text {des }}$ to the same speed $v_{\text {des }}=1.34(\mathrm{~m} / \mathrm{s})$ and obey the normal distribution $v_{\text {des }} \sim N\left(1.34,0.1^{2}\right)(\mathrm{m} / \mathrm{s})$. The transition probability of pedestrians walking or stopping at each step is:

$$
P_{m}=v_{\text {des }} / v_{\max } \cdots P_{s}=1-v_{\text {des }} / v_{\max }
$$

In the formula, $P_{m}$ is the probability of pedestrian movement, $P_{s}$ is the probability of pedestrian stopping, that is, the probability of pedestrian moving at each step is $v_{\text {des }} / v_{\text {max }}$.

When the expected speed is the same, each pedestrian has a probability of walking of 0.667; at the same time, because pedestrians can go in the oblique direction, the actual speed of pedestrians is still heterogeneous, and it can be approximated that pedestrians have slight speed heterogeneity. However, there is no heterogeneity in the prediction range. For pedestrians with different expected speeds, the probability of walking in each walk is different, thereby achieving completely random movement. In this study, the pedestrian expected speed distribution obeys a normal distribution with a smaller variance (compared to the general setting $v_{\text {des }} \sim N\left(1.34,0.34^{2}\right)(\mathrm{m} / \mathrm{s})$, which is due to the fact that the pedestrians are symmetrical in a circle. The walking experiment is required to walk as fast as possible (running is not allowed).

\subsection{Predict Field Potential Energy and Its Heterogeneity}

According to the calculation method of predicted field potential energy, we expand it accordingly and apply it to the subdivided cellular automata model. The field potential energy (anticipation floor field) value represents the expected value of pedestrians arriving in the future cell along the desired direction. The calculation is determined by the reserved 
cell and the direction of the pedestrian. For this reason, the direction of the pedestrian is extended to eight directions, that is, up, top right, top, top left, left, bottom left, bottom, and bottom right.

The reserved cells represents the cell that the pedestrian will pass in the current direction in the future. In the traditional cell, the non-negative integer parameter $d_{A}$ is used to indicate the pedestrian's prediction range, but in the subdivided cell, its non-negative integer parameter is $d_{A}$. This is related to the expected speed of pedestrians, that is, it also reflects the heterogeneity of pedestrians, adjusted to $d_{A}=n * v_{\text {des }} \cdot t_{A} / a$. The reserved cells for the eight directions of pedestrians are described by the straight direction and the diagonal direction as representatives. The schematic diagram is shown in Figures 4 and 5.

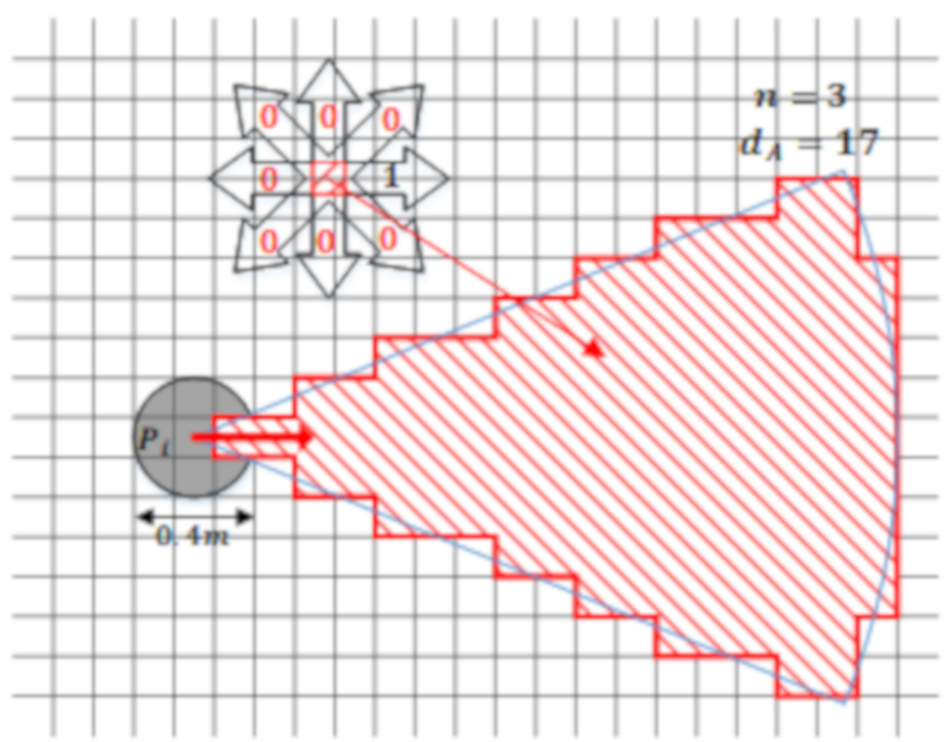

Figure 4. Schematic diagram of reserved cells for pedestrians on the right.

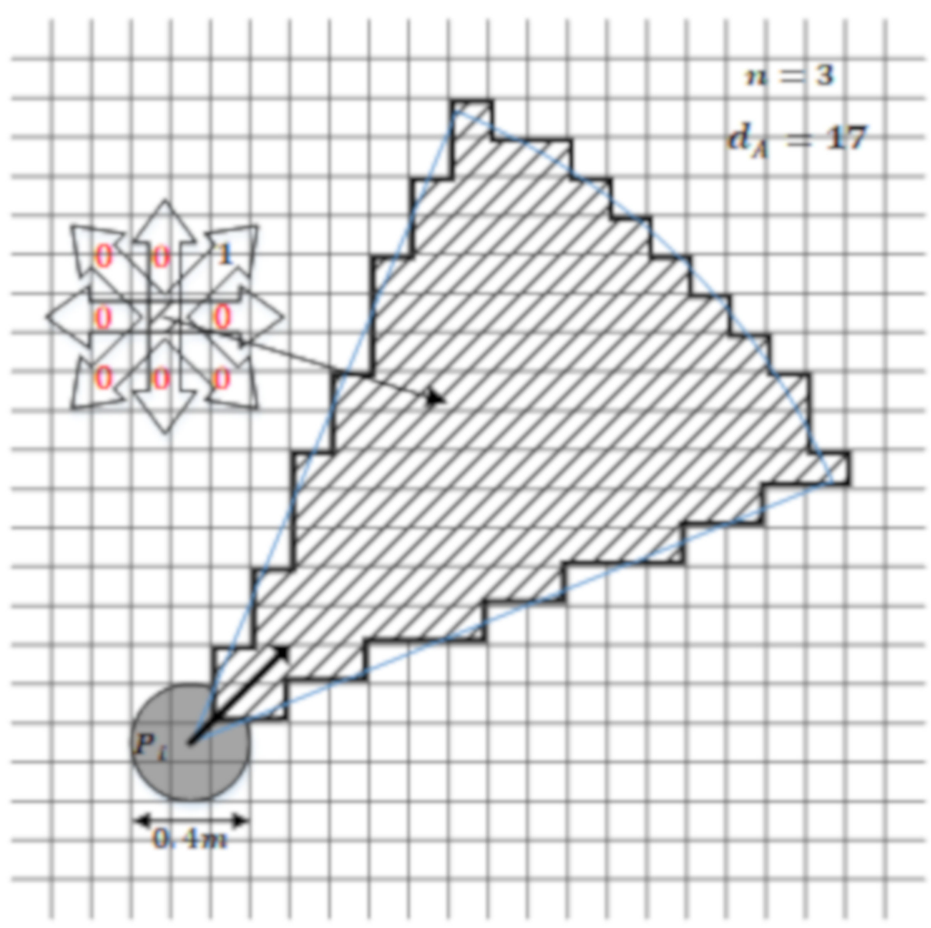

Figure 5. Schematic diagram of reserved cells for pedestrians on the upper right. 
According to the reserved cells, the value of the predicted field potential energy can be calculated. The predicted field potential energy (AFF) is described by the non-negative integer $A_{i j}^{(n)}$, where $n \in\{1,2,3,4,5,6,7,8\}$, corresponding to eight directions, that is, 1 corresponds to right, 2 corresponds to upper right, 3 corresponds to upper, 4 corresponds to upper left, 5 corresponds to left, 6 corresponds to lower left, 7 corresponds to lower, and 8 corresponds to bottom right, see Figure 6.

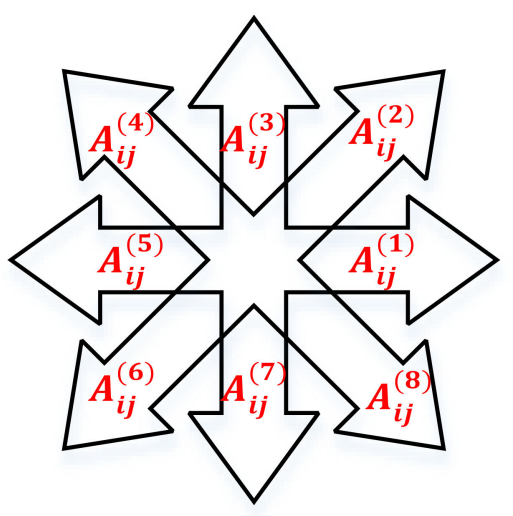

Figure 6. $A_{i j}^{(n)}, n=1,2,3,4,5,6,7,8$ The relationship between the value and the direction.

For the same cell, its predicted field potential energy has eight different values. At the same time, the calculation of $A_{i j}^{(n)}, n=1,2,3,4,5,6,7,8$ is determined by the number of reserved cells and the direction, and the following steps are followed.

Step 1: If the cell is a reserved cell that a pedestrian expects to arrive in the future along the current direction, the AFF in the direction of the reserved cell plus one, that is, $A_{i j}^{(n)}=A_{i j}^{(n)}+1, n=1,2,3,4,5,6,7,8$ (see Figures 4 and 5). Here, we further use two pedestrians as an example to describe the relationship between the pedestrian's reserved cells and the AFF value. In Figure 7, the pedestrian $P_{i}$ walks in the upper right direction, and the pedestrian $P_{j}$ walks in the upward direction. The reserved cells of the two persons overlap partly. The blue box in Figure 7 shows if the cell is the reserved cell of two persons. For each cell in the line, the predicted field value is added to the right and up-right directions of these cells, namely $A^{(1)}=1$ and $A^{(2)}=1$. The other reserved cells are added by one in the upper right and upper directions, respectively, as shown in Figure 7, for each reserved cell in the red and black borders.

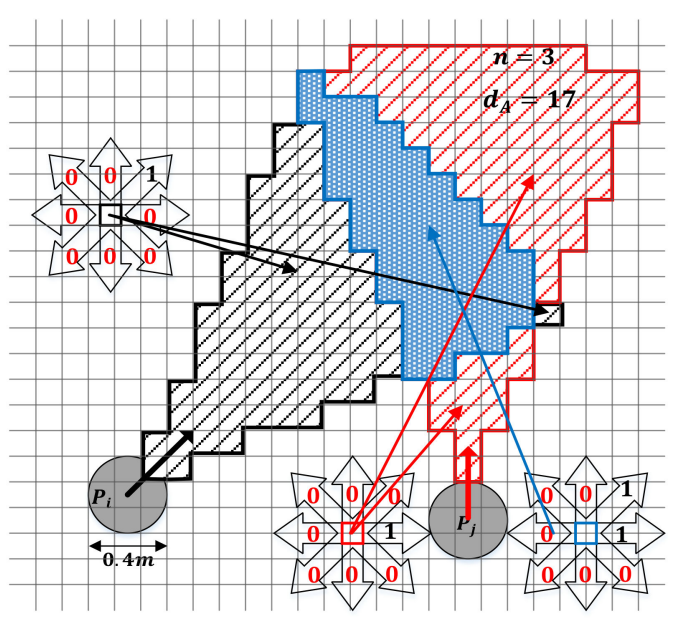

Figure 7. AFF value and calculation method of reserved cells. 
Step 2: Calculate $E_{i j}^{(n)}, n=1,2,3,4,5,6,7,8$ by predicting the field potential energy $A_{i j}^{(n)}, n=1,2,3,4,5,6,7,8$ (see Formula (4)).

$$
E_{i j}^{(n)}=\sum_{n^{\prime} \neq n} A_{i j}^{\left(m^{\prime}\right)}
$$

Here, $\mathrm{n}$ corresponds to the pedestrian's direction at cell $(i, j)$. It should be noted that $E_{i j}^{(n)}$ is different for each pedestrian. In other words, $A_{i j}^{(n)}$ is a global value, and $E_{i j}^{(n)}$ is not a global value. When the value of $E_{i j}^{(n)}$ in the direction points to $n$ on $(i, j)$, the AFF values in the direction except for the n direction should be summed. The value of $E_{i j}^{(n)}$ represents the subjective impression that the cell $(i, j)$ will be occupied in the future.

\subsection{Steering Equation}

In the subdivided cellular automata model, the direction of pedestrian movement is determined according to the static field $S_{i j}$ and the dynamic field $D_{i j}$. In this study, we choose eight directions, namely right, up right, up, up left, left, down left, down, down right, and stop. To the direction $\vec{\lambda}_{m}$, m represents right, up right, up, up left, left, down left, down, down right, and stop. The turning probability is:

$$
P\left[\vec{\lambda}(t)=\vec{\lambda}_{m}\right]=N \xi_{i j} \exp \left(-k_{A} E_{i j}^{(n)}\right) \exp \left(k_{S} S_{m j}\right) \exp \left(k_{D} D_{m j}\right)
$$

In the formula, $\mathrm{N}$ is the regularization coefficient to ensure $\sum p_{i j}=1$.

$$
\xi_{i j}=\left\{\begin{array}{cc}
1, & \text { cell }(i, j) \text { is not occupied by obstacles } \\
0, & \text { otherwise }
\end{array}\right.
$$

$\xi_{i j}$ represents the influence of obstacles, $k_{S}$ and $k_{D}$ are the sensitivity parameters of the static field potential energy and the dynamic field potential energy of the scene, respectively. Here, $k_{S}$ reflects the straightness of pedestrians towards the goal, and $k_{D}$ reflects the tendency of pedestrians to foll. Steering equation

\subsection{Conflict Resolution}

According to the repelling volume effect, it is not allowed for pedestrians to move toward a certain cell to occupy the volume of other pedestrians. In the cellular automata model, the position of pedestrians is updated synchronously, and multiple pedestrians will compete for the same cell. Therefore, conflict resolution methods need to be proposed to deal with this situation. One solution is for pedestrians to randomly win the competition, while other pedestrians return to their original cell positions. Another solution is to choose the pedestrian's expected speed as an indicator. Pedestrians with higher expected speed are not easy to stop suddenly, so it is easier to win the conflict, and other pedestrians lose the competition and return to the original cell. As shown in Figure 8.

In addition, for the subdivided cells, according to the principle of exclusion volume, the conflict situation of pedestrians will change accordingly. Since pedestrians with higher expectations are less willing to stop and let others pass, they are more aggressive in conflicts. The probability of pedestrian $P_{i}$ winning the competition is

$$
\mu=\left\|\vec{v}_{i}\right\|^{k} / \sum_{i}\left\|\vec{v}_{i}\right\|^{k}
$$

In the formula, $\mathrm{k}$ reflects the average competitiveness of pedestrians participating in the conflict. If $k=0$, pedestrians participating in the conflict have the same competitiveness even if they have different expected speeds. With $k \rightarrow \infty$, only the pedestrian corresponding to the maximum expected speed can move to the target cell. 


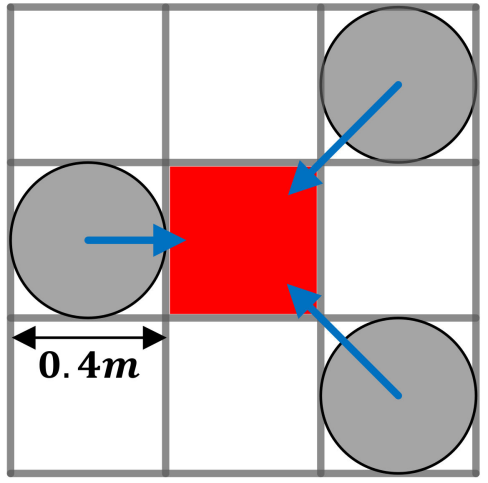

(a)

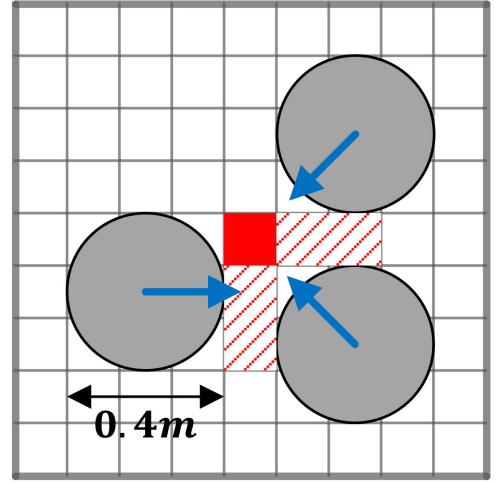

(b)

Figure 8. AFF value and calculation method of reserved cells. (a) Schematic diagram of traditional cell conflict; (b) Schematic diagram of subdivided cell conflict.

\subsection{Simulation Update Program}

In the update of pedestrian cell position, according to the principle of exclusion volume, pedestrians cannot occupy the cells occupied by other pedestrians, that is, the cells of pedestrians cannot overlap. Therefore, on the basis of the synchronous update rules, that is, the corresponding conflict resolution method, we make corresponding changes to add the influence of the prediction field.

When the simulation time $t=0$, the static field $S_{i j}$ is calculated, and at the same time, $D_{i j}$ is set to zero for all cells. In the process of $t \rightarrow t+1$, the simulation update procedure of the field potential energy model is as follows.

Step 0.

(A) The value of AFF in different directions is reset to zero, see the formula $A_{i j}^{(1)}=$ $A_{i j}^{(2)}=A_{i j}^{(3)}=A_{i j}^{(4)}=A_{i j}^{(5)}=A_{i j}^{(6)}=A_{i j}^{(7)}=A_{i j}^{(8)}=0$.

(B) Obtain the reserved cell based on the current position of all pedestrian cells and the walking direction, and then obtain the value of $\operatorname{AFF} A_{i j}^{(n)}, n=1,2,3,4,5,6,7,8$.

(C) Calculate $E_{i j}^{(n)}, n=1,2,3,4,5,6,7,8$ for each pedestrian.

Step 1 . The pedestrian decides to move to his feasible neighbor cell according to the moving probability, and updates the cell position of the pedestrian.

Step 2. Determine whether the pedestrian has a conflict. If yes, go to Step 3; if not, go to Step 4.

Step 3. Resolve the conflict and enter Step 4.

Step 4. If the pedestrian leaves the currently occupied cell, the dynamic field potential value $D_{i j}$ of the current cell is increased by one. Otherwise, the dynamic field potential energy value $D_{i j}$ remains unchanged.

Step 5. All $D_{i j}$ expand with probability $\delta$ and dissipate with probability $\alpha$.

\section{Scene Setting and Simulation}

The circle antipode experiment was implemented in the open space in front of the Beijing Jiaotong University teaching building in 2017. The experiment was divided into two types with a radius of $5 \mathrm{~m}$ and $10 \mathrm{~m}$, and each semicircle is uniformly marked with No. 1-32 (that is, the symmetrical position is the same number, see Figure 9). In this experiment, the total number of participants was 64 ( 31 women, 33 men), all of whom were students from Beijing Jiaotong University, aged 18-28. All participants were required to wear shady clothes and wear hats of different colors according to their heights for the subsequent extraction of video data. Blue, red, and yellow correspond to height ranges of 155-165 cm, $165-175 \mathrm{~cm}$, and $175-185 \mathrm{~cm}$, respectively. 


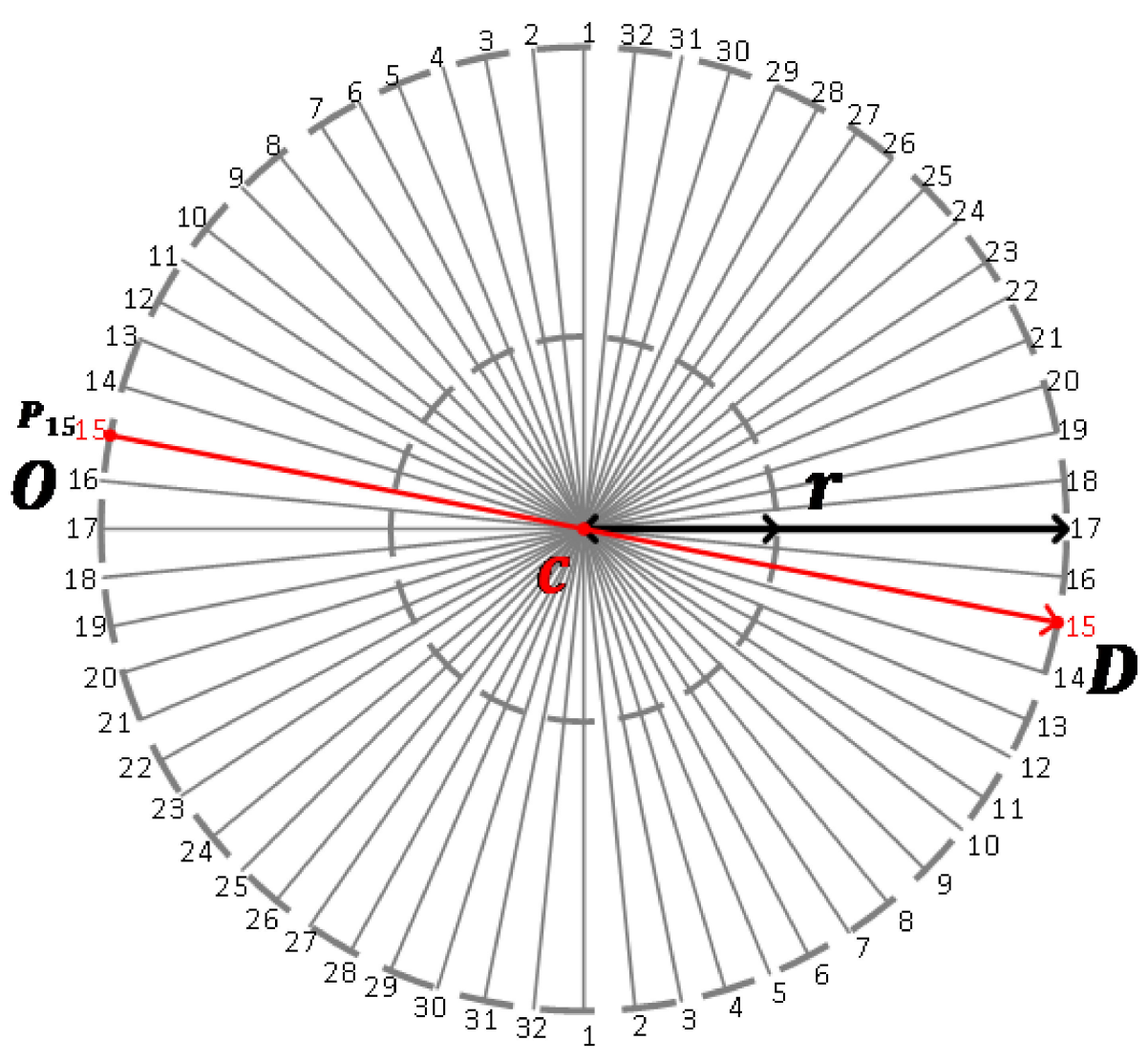

Figure 9. Overview of the experimental scene.

The number of participants in experiments with different radiuses included 8, 16, 32, and 64 experiments. Each experiment was repeated four times. In each experiment, the total number of participants was divided into two groups AB. Taking the 10-m experiment involving 64 people as an example, 64 people were randomly divided into two groups $A B$, 32 people in each group, and a number from 1 to 32 was assigned. At the beginning of the experiment, the two groups of people occupy a semicircle. After receiving the start password, all pedestrians set off at the same time and walked toward their symmetrical positions, that is, on the punctuation of the same number. In addition, all pedestrians were required to walk to the target point as quickly as possible while ensuring their own safety, but it is not recommended to run to the target point. A high-definition camera located above the experimental scene was used to record the video data of the experiment, and the number of video frames was 25 frames per second.

\subsection{Scene Setting}

In the traditional field potential energy model, the extended predicted field potential energy was introduced and the cell size was subdivided. First, it can reflect the pedestrian's predicted detour behavior in the circular symmetrical walking experiment, and pedestrians share the reserved cell representative The common conflict area, perception of common conflict and behavior to avoid conflict are the main characteristics of pedestrian circumvention behavior in this scene. Second, it can describe the distribution position of pedestrians in a more detailed manner. In the circular symmetrical walking experiment, the refined location description of pedestrians is closer to reality, which is crucial to the effect of simulation.

In the circular symmetry experiment, pedestrians were evenly distributed on a circle with a radius of $\mathrm{R}=5,10(\mathrm{~m})$, and the scene size was set to $\left.2 R \times 2 R\left(\mathrm{~m}^{2}\right)\right)$. Under a given subdivision $\mathrm{n}$, the cell size was subdivided to $0.4 / n(\mathrm{~m})$; thus, the scene was composed of $5 * R * n \times 5 * R * n$ cells. Figure 10 shows the initial pedestrians when the subdivisions are 
$\mathrm{n}=3$ and $\mathrm{n}=5$. Location distribution. It can be seen from the figure that, as the subdivision $\mathrm{n}$ increases, the cell size becomes smaller and smaller, so that the position distribution of pedestrians is more in line with the actual scene.

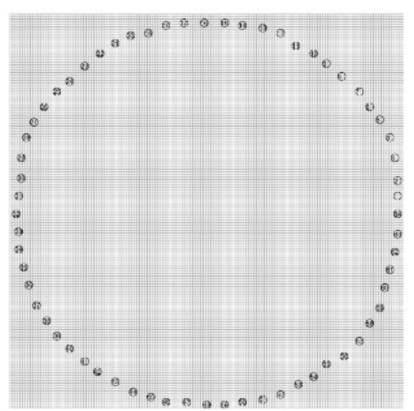

(a)

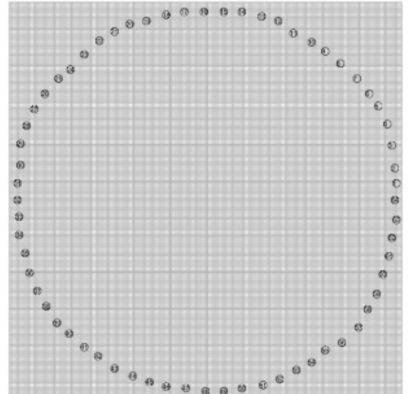

(b)

Figure 10. Pedestrian's initial position distribution map. (a) when subdivision $\mathrm{n}=3$; (b) when subdivision $\mathrm{n}=5$.

\subsection{The Impact of Subdivision and Heterogeneity on Simulation}

In the simulation analysis of this experiment, the third experiment of $64 \mathrm{p}-10 \mathrm{~m}$ was also selected as the simulation object to explore the effect of the subdivision of the cell and the field potential energy on the simulation.

Considering the limitation of the degree of subdivision on computer calculation speed, we chose the degree of subdivision $n=1,3,5,7,9,11,13$, and 15 . To reflect the heterogeneity of pedestrian speed, the expected speed distribution of pedestrians is $v_{d e s} \sim N\left(1.34,0.1^{2}\right)(\mathrm{m} / \mathrm{s})$ or the same speed is $v_{\text {des }}=1.34$; the time step is $\Delta t=0.4 / n \cdot v_{\max }(\mathrm{s})$; the corresponding maximum speed is $v_{\max }=2(\mathrm{~m} / \mathrm{s})$; the prediction range parameter is $d_{A}$; the prediction field is $E_{i j}^{(n)}$; the weight value, also described as heterogeneity, is $k_{A} ; d_{A}$ is related to the expected speed of pedestrians, as $d_{A}=n * v_{d e s} \cdot t_{A} / a ; k_{A}$ is related to $d_{A} ; k_{A}=d_{A} / 6 \cdot n$, the static field $S_{i j}$ of the cellular automata model of the field potential energy; and the dynamic field $D_{i j}$ parameters are the same under different subdivisions. See the parameter settings Table 1.

Table 1. Parameter setting table of cellular automata model.

\begin{tabular}{cccc}
\hline Parameter & Value & Parameter & Value \\
\hline$k_{S}$ & 5 & $n$ & $1,3,5,7,9,11,13,15$ \\
$k_{D}$ & 0.2 & $v_{\text {des }}$ & $\left(1.34,0.1^{2}\right)(\mathrm{m} / \mathrm{s})$ \\
$k_{A}$ & $d_{A} /(6 \cdot n)$ & $\Delta t$ & $0.4 /\left(n \cdot v_{\text {max }}\right)(\mathrm{s})$ \\
$d_{A}$ & $n \cdot v_{d e s} \cdot t_{A} / a$ & & \\
\hline
\end{tabular}

The cumulative density of the simulation scene reflects the density distribution characteristics of pedestrians, and can effectively reflect the area where pedestrians gather. The calculation formula of the cumulative density at the cell $(i, j)$ is shown in Equation $(8)$.

$$
C D_{i, j}=\sum_{t=1}^{T} \delta_{i, j}^{t}
$$

In the formula, $\mathrm{t}$ is the current time step, $\mathrm{T}$ is the total time of the simulation, and $\delta_{i, j}^{t}$ is the state of the cell $(i, j)$ at time $t$, namely

$$
\delta_{i, j}^{t}=\left\{\begin{array}{lc}
1, & \text { cell }(i, j) \text { iss occupied at time } \\
0, & \text { otherwise }
\end{array}\right.
$$


Pedestrians are described as homogeneous (that is, the expected speed and prediction range of pedestrians are the same). The cumulative probability density diagram obtained by the experimental simulation of circular symmetrical walking under different subdivisions under the field potential energy cellular automata model is shown in Figure 11.

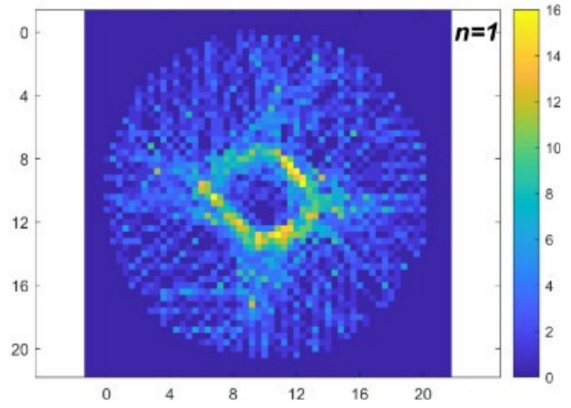

(a)

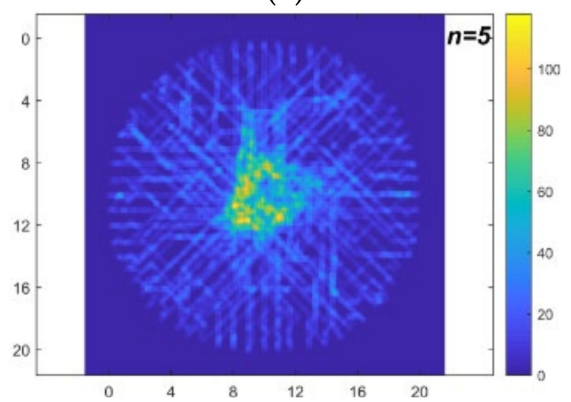

(d)

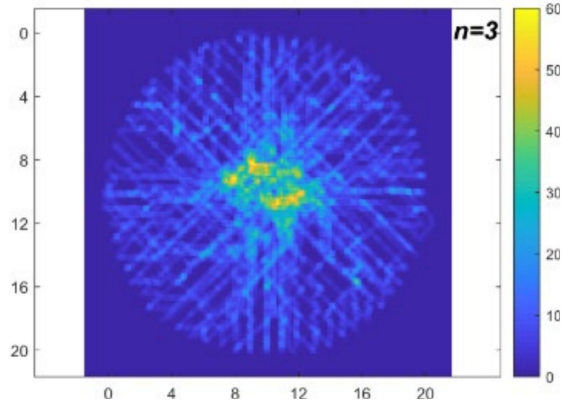

(b)

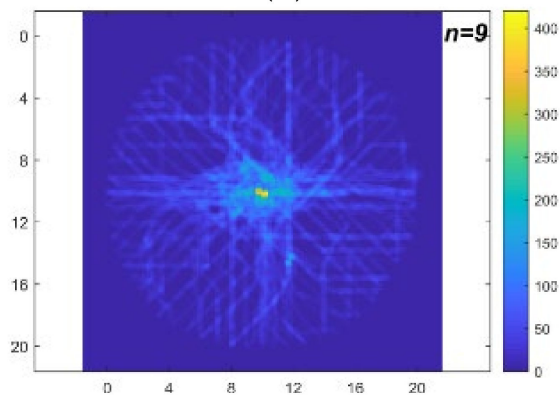

(e)

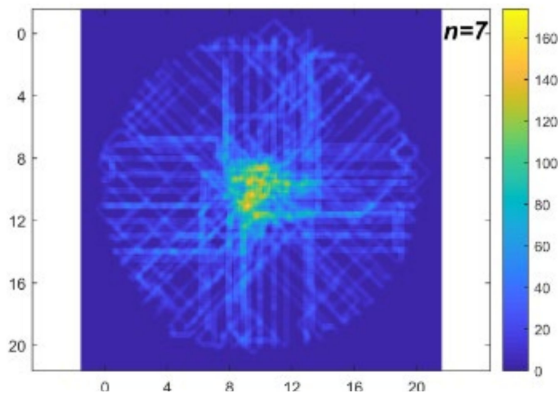

(c)

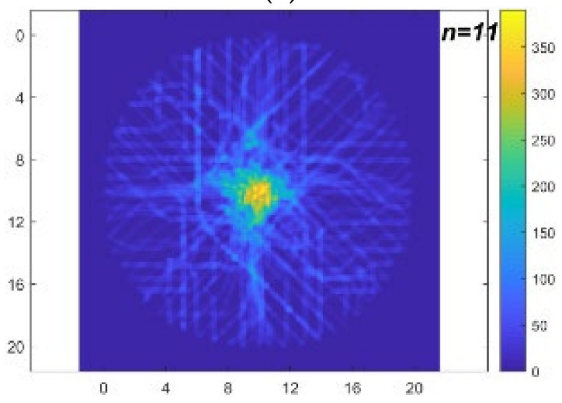

(f)

Figure 11. Pedestrian cumulative density map under each subdivision (no heterogeneity). (a) when subdivision $\mathrm{n}=1$; (b) when subdivision $\mathrm{n}=3$; (c) when subdivision $\mathrm{n}=5$; (d) when subdivision $\mathrm{n}=7$; (e) when subdivision $\mathrm{n}=9$; $(\mathbf{f})$ when subdivision $\mathrm{n}=11$.

It can be seen from Figure 10 that the cumulative density of the central area is larger than that of other areas, and, at the same time, the characteristics of pedestrian trajectory distribution can be seen. This shows that homogeneous pedestrians all walk to the central area at the same time (approximately), gather in the central area, and then walk towards their respective target cells. Pedestrians are described as field potential energy cellular automata model under heterogeneity (velocity, prediction range). The cumulative density map obtained by experimental simulation of circular symmetric walking under different subdivisions is shown in Figure 12.

It can be seen from Figure 11 that, due to the heterogeneity of pedestrians, the cumulative density of the central area presents an obvious ring shape, which shows that pedestrians with high expected speed have a larger prediction range, so it will first judge that the central area is about to happen. The area is crowded, thus it receives the influence of the forecast field and makes decisions to go in other directions to avoid congestion, and pedestrians present a unified detour pattern.

In addition, the degree of subdivision refines the walking speed of pedestrians. For this reason, we have studied the change graph of the average speed of pedestrians in each subdivision, as shown in Figure 13. 


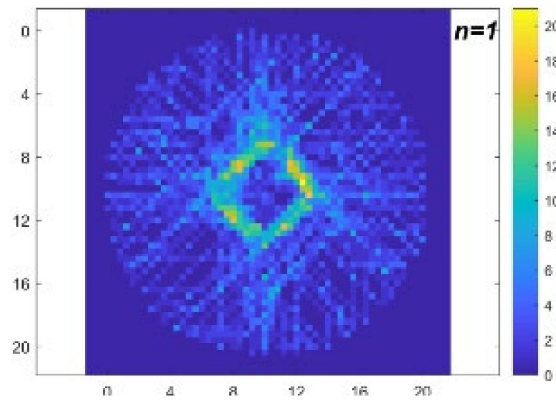

(a)

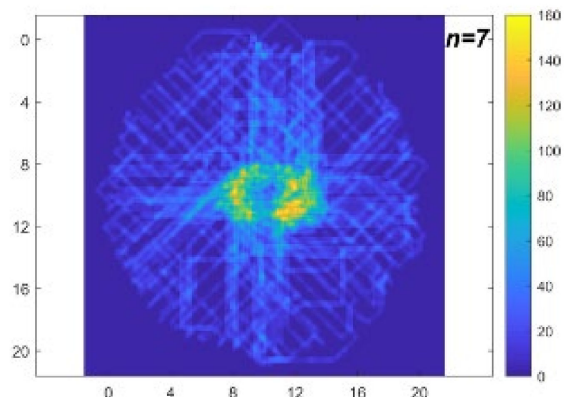

(d)

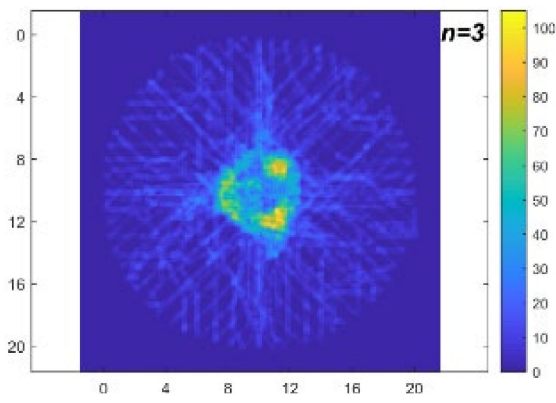

(b)

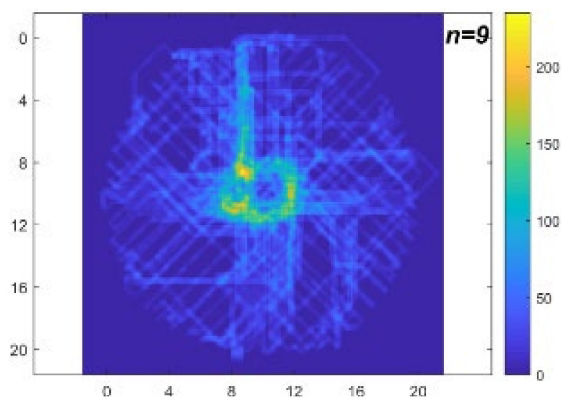

(e)

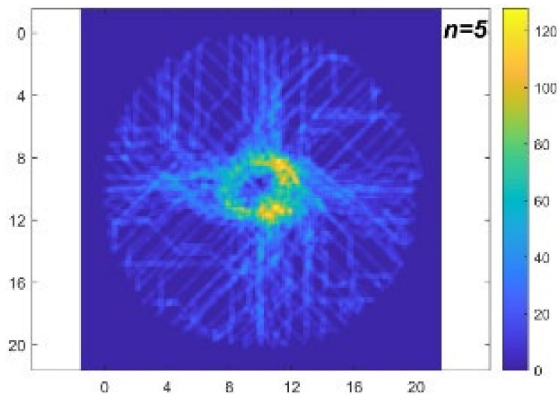

(c)

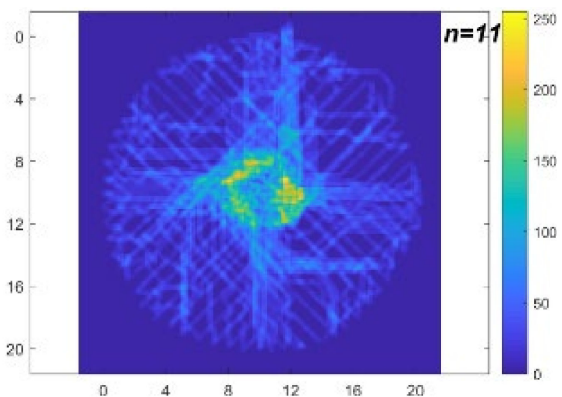

(f)

Figure 12. Pedestrian cumulative density map under each subdivision (heterogeneity). (a) when subdivision $n=1 ;(\mathbf{b})$ when subdivision $\mathrm{n}=3$; (c) when subdivision $\mathrm{n}=5 ;(\mathbf{d})$ when subdivision $\mathrm{n}=7$; (e) when subdivision $\mathrm{n}=9$; $(\mathbf{f})$ when subdivision $\mathrm{n}=11$.

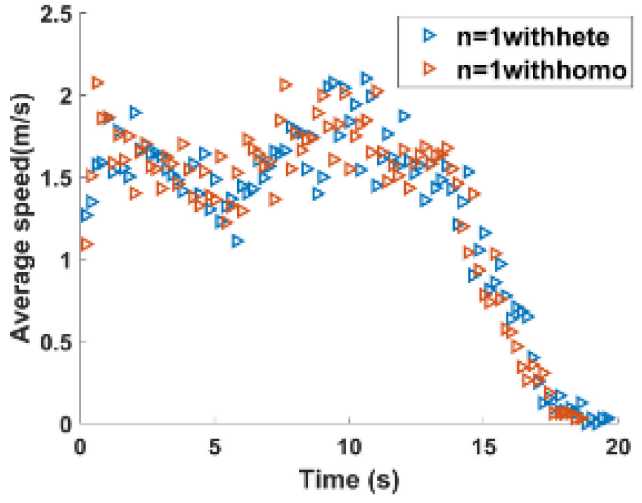

(a)

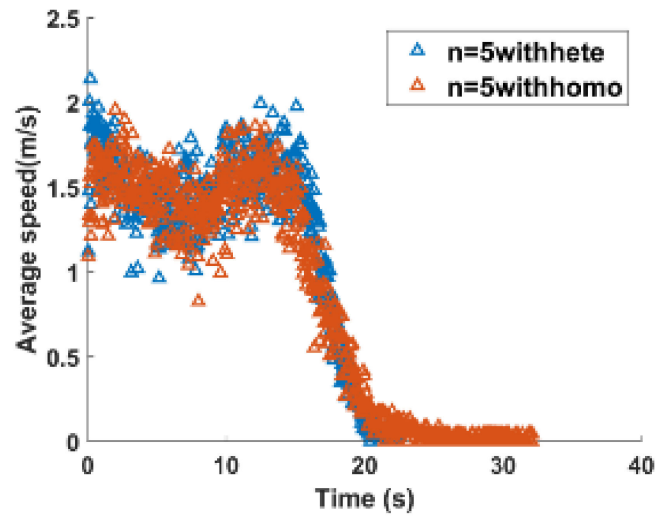

(c)

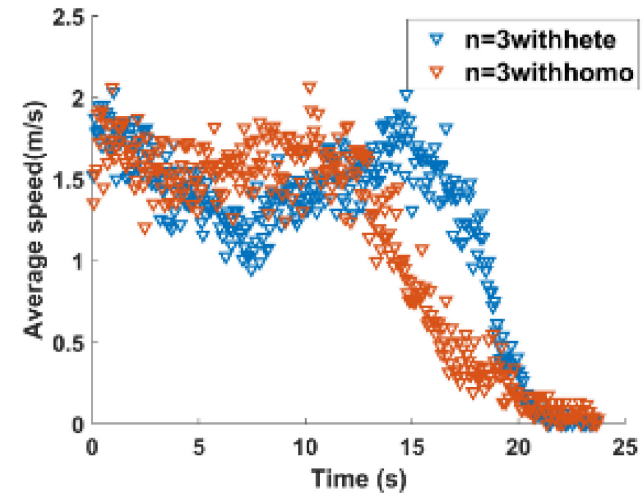

(b)

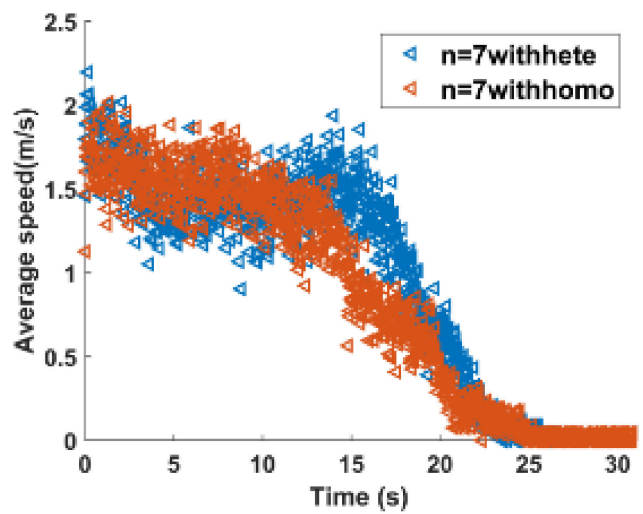

(d)

Figure 13. The average speed change graph for the presence or absence of heterogeneity of pedestrians in each subdivision. (a) when subdivision $\mathrm{n}=1$; (b) when subdivision $\mathrm{n}=3$; (c) when subdivision $\mathrm{n}=5$; (d) when subdivision $\mathrm{n}=7$. 
It can be seen from Figure 13 that, when the degree of subdivision $n=1$, there is no significant difference in the average speed change in pedestrians with or without heterogeneity, and, as the degree of subdivision $n$ increases, the gap between whether or not pedestrians is heterogeneous begins to change. When $n=3,7$, the average pedestrian speed of homogeneity almost remains almost unchanged when the conflict occurs, while the average speed of heterogeneous pedestrians decreases due to the occurrence of congestion, and then increases, showing a double peak. Change trend; in fact, the average speed changes of heterogeneous pedestrians all show the change in double peaks, while the change in homogeneous pedestrian speed is significantly smaller than that of homogeneous pedestrians. The subdivision $n=3,7$ shows two types of simulation. Homogeneous pedestrian average speed changes did not show double peak changes. This is because the pedestrian speed change range is limited by the degree of subdivision and the probability of each step of the movement. The larger the subdivision $n$, the smaller the time step, that is, the smaller the step frequency. Pedestrians walk in accordance with the probability, resulting in a different number of pedestrians choosing to walk at each step, so the average speed change is smaller than that of heterogeneous pedestrians. In addition, in each subdivision, the average speed of the heterogeneous pedestrians is compared with the homogeneous pedestrians. The time when the average speed decreases is the time when the common center conflict occurs, and the average pedestrian speed increases again when the pedestrian has finished processing the center. After the area, walk to the target cell again at the desired speed.

At the same time, the change in the average distance from the center also reflects the movement state of pedestrians during the movement, indicating the overall decisionmaking of pedestrians to cope with the congestion in the central area. The average distance of pedestrians from the center under different subdivisions of homogeneity and heterogeneity is analyzed. The average distance change diagram of the simulation of homogeneous pedestrians from the center is shown in Figure 14. The corresponding average distance of the simulation of heterogeneous pedestrians from the center is shown in Figure 14. The change diagram is shown in Figure 15.

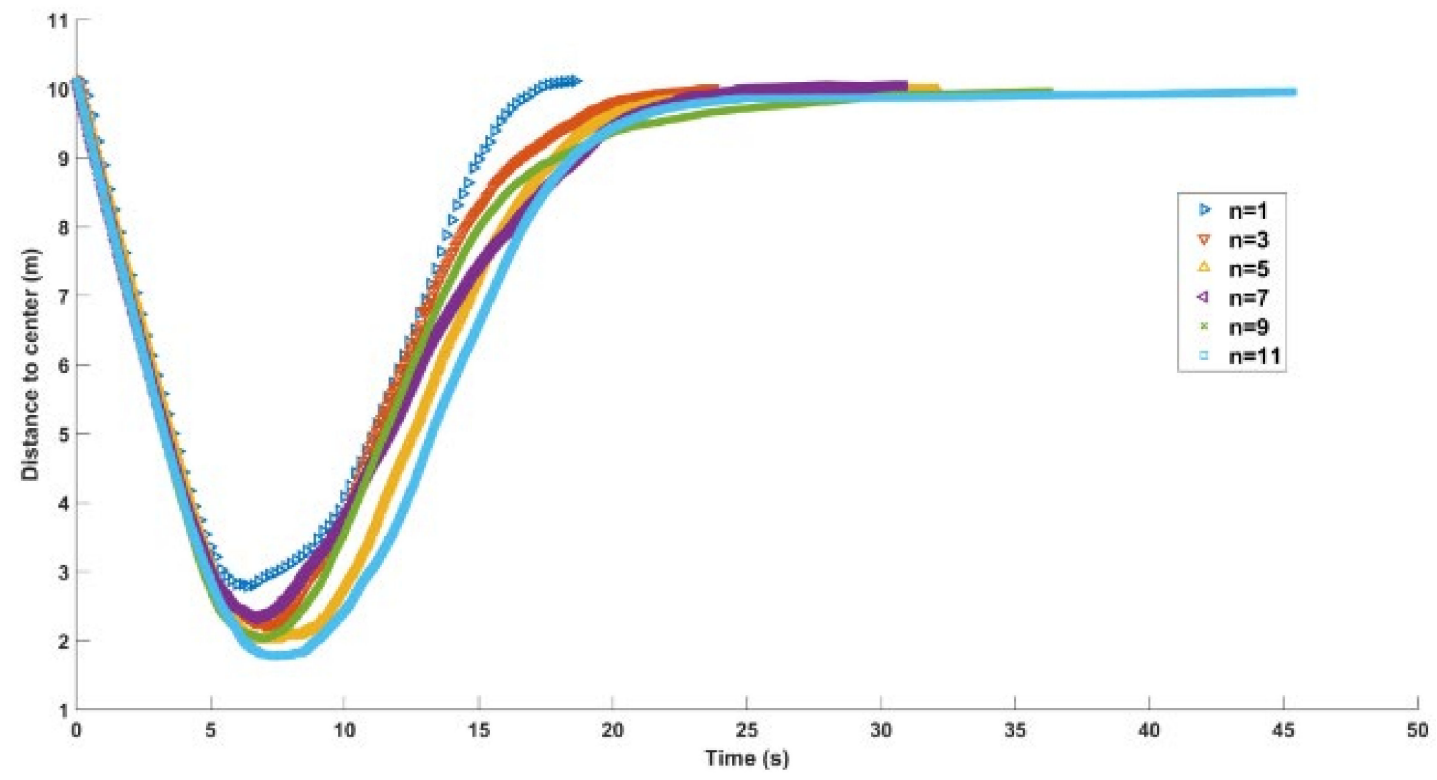

Figure 14. The change in distance from the center of the homogeneity of pedestrians with different subdivisions. 


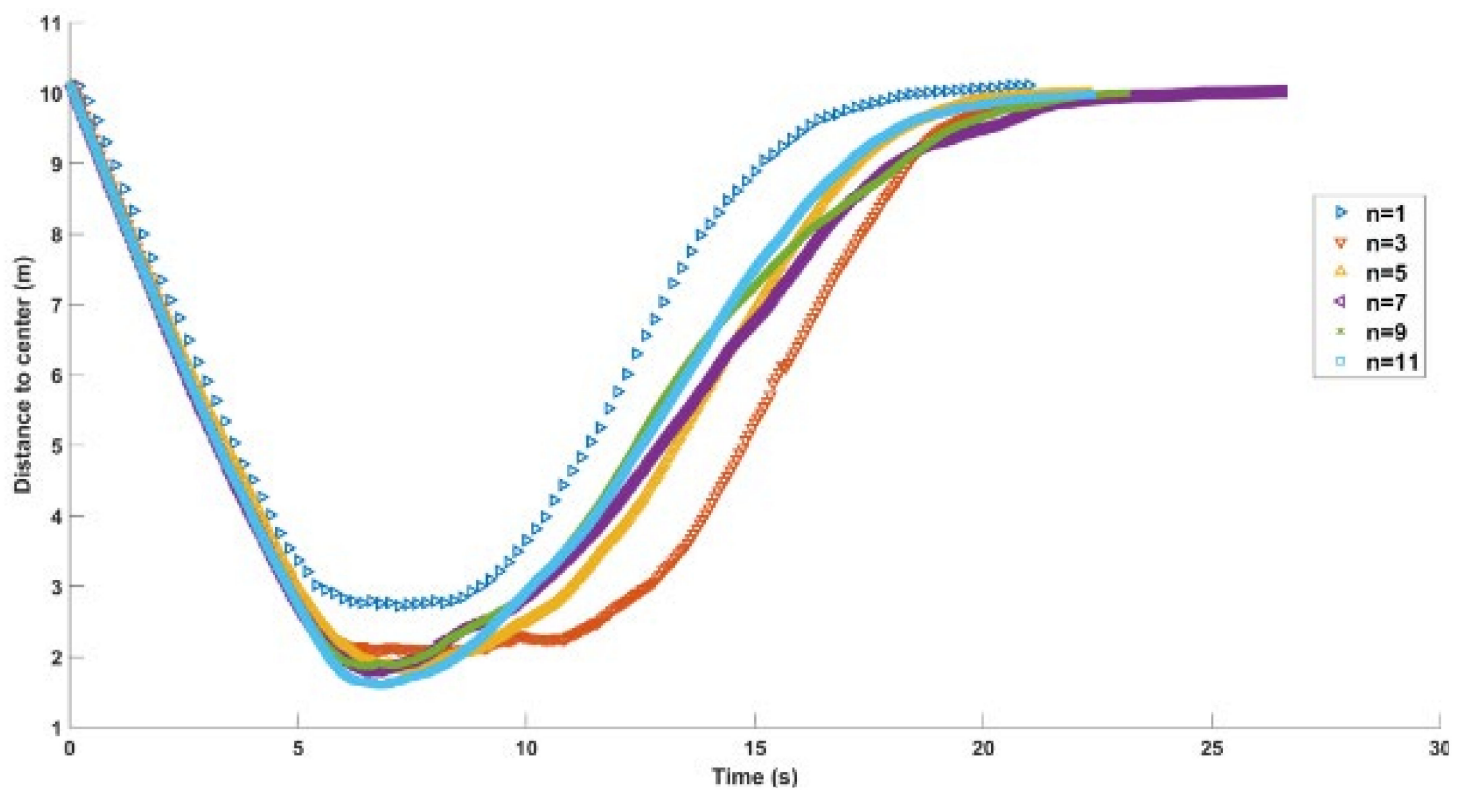

Figure 15. The change in the distance from the center of the pedestrian heterogeneity of different subdivisions.

It can be seen from Figures 14 and 15 that the change in the average distance between pedestrians and the center presents a uniform feature, that is, a U-shape. The moment of the minimum average distance from the center represents the maximum degree of congestion and is about to dissipate. In addition, the comparison chart of the average distance change between homogeneous and heterogeneous pedestrians from the center under different subdivisions is shown in Figure 16.

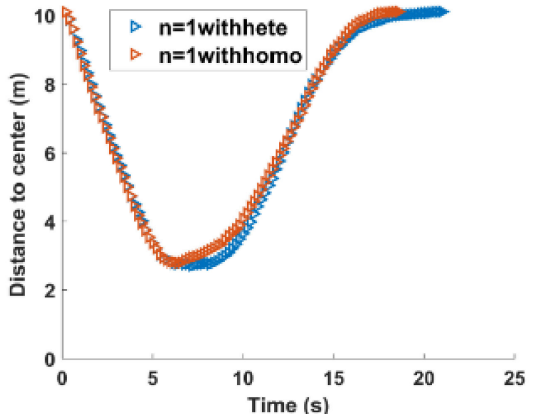

(a)

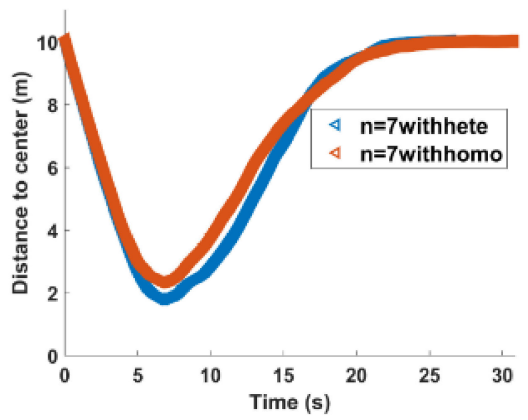

(d)

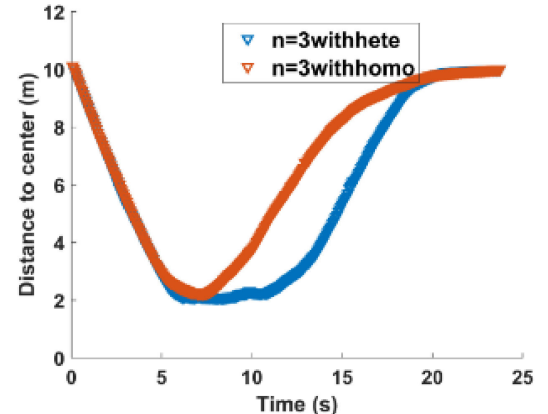

(b)

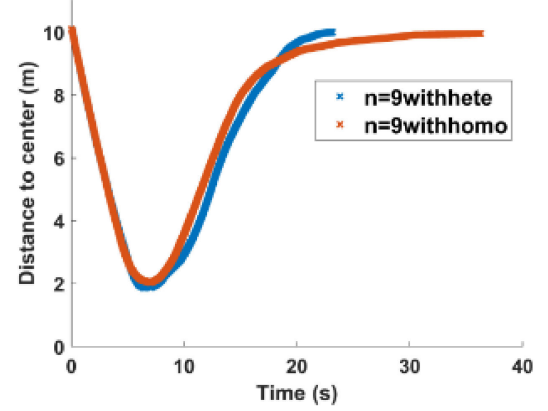

(e)

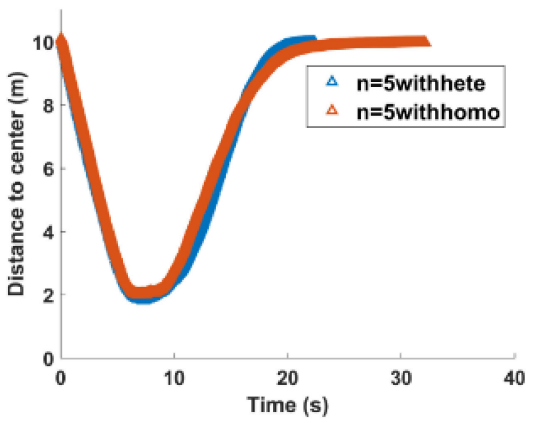

(c)

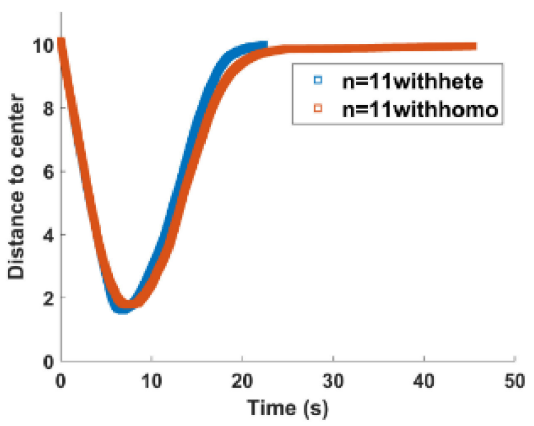

(f)

Figure 16. Different subdivisions of pedestrians with or without the heterogeneity of the distance change from the center. (a) when subdivision $\mathrm{n}=1$; (b) when subdivision $\mathrm{n}=3$; (c) when subdivision $\mathrm{n}=5$; (d) when subdivision $\mathrm{n}=7$; (e) when subdivision $\mathrm{n}=9$; $(\mathbf{f})$ when subdivision $\mathrm{n}=11$. 
It can be seen from Figure 16 that the time for heterogeneous pedestrians to stay at the minimum is slightly longer than that of homogeneous pedestrians. Heterogeneous pedestrians cause the congestion resolution time to be slightly slower than that of homogeneous pedestrians, that is, it is easier for homogeneous pedestrians. Congestion should be quickly removed and the target point at a faster average speed should be moved towards.

\subsection{Predict the Influence of Field Potential Energy AFF on Simulation}

The rough analysis also includes the influence of the predicted field potential energy on the simulation. Through the comparison and analysis of the predicted field potential energy model under the same subdivision and the same parameters with the traditional field potential energy, including the pedestrian cumulative density map, pedestrian trajectory, and average speed change analysis. As shown in Figure 16, when $n=1$, there is a comparison of the cumulative density of the predicted field potential energy AFF.

It can be seen from Figures 17 and 18 that, after adding the predicted field potential energy AFF, the pedestrian gathering area turns from the central area to the near-central area, and presents a ring shape. This shows that the existence of the prediction field makes pedestrians stop near the center and move in other directions to get rid of the congestion in the center. Correspondingly, the 1, 15, 30, 45, and 60 pedestrians in the simulation are selected for comparison, and the pedestrian trajectory comparison chart is shown in Figure 19.

As can be seen from Figures 19 and 20, i.e., the pedestrian trajectory diagram (Figure 19a,c,e,g,i and Figure 20a,c,e,g,i), the same pedestrian trajectory diagram with AFF added (Figure 19b,d,f,h,j and Figure 20b,d,f,h,j) obviously has a larger detour path when encountering congestion in the central area. AFF can make pedestrians go in other directions to avoid congested areas, and can better reflect pedestrians' detour behavior in common conflict areas.

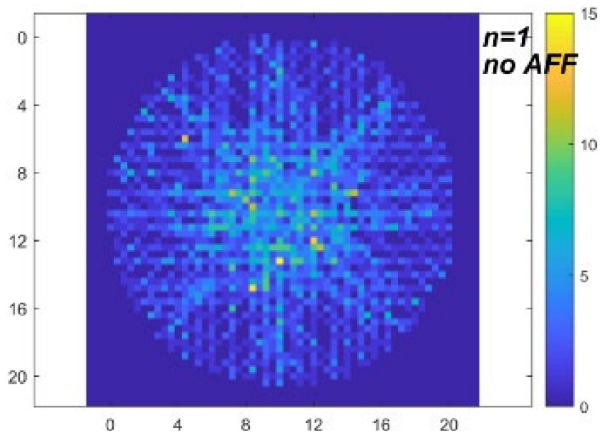

(a)

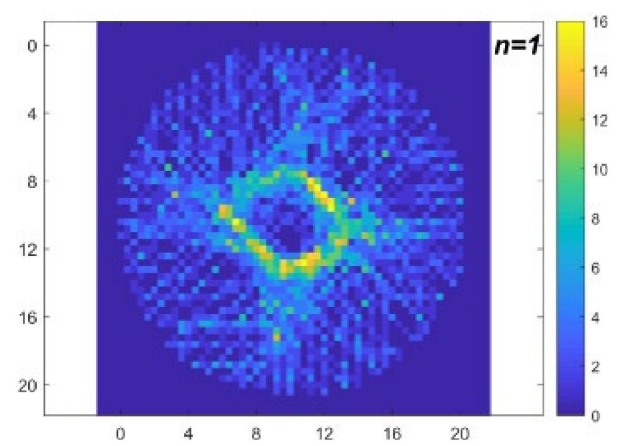

(b)

Figure 17. Contrast chart of homogeneous pedestrian cumulative density with or without predicted field potential energy AFF. (a) No prediction field; (b) There is a prediction field.

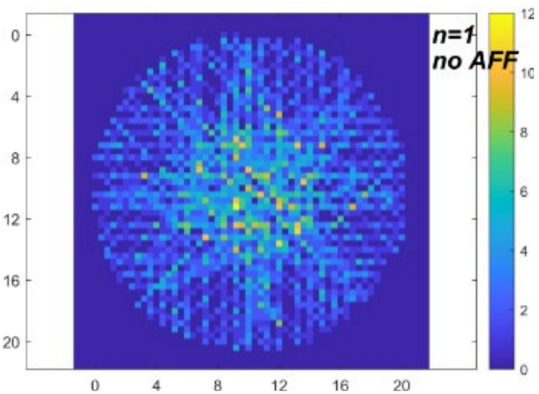

(a)

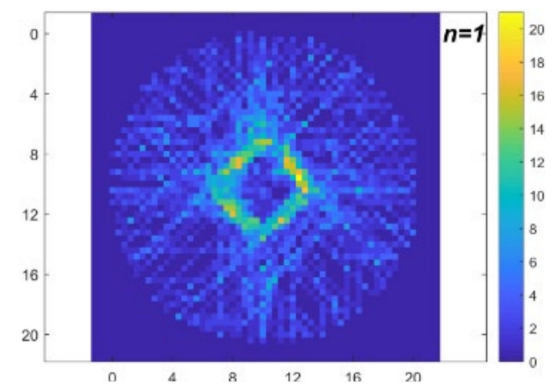

(b)

Figure 18. Comparison of cumulative density of heterogeneous pedestrians with or without predicted field potential energy AFF. (a) No prediction field; (b) There is a prediction field. 


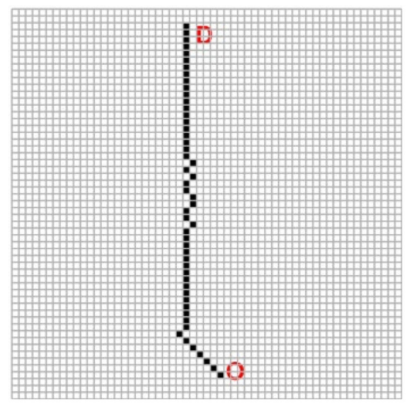

(a)

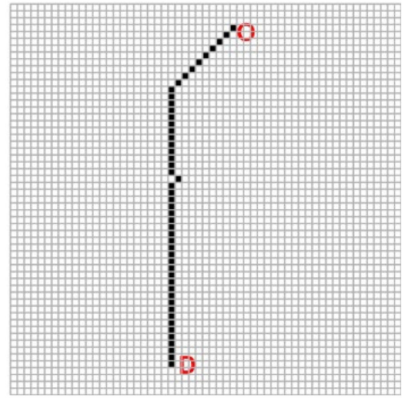

(e)

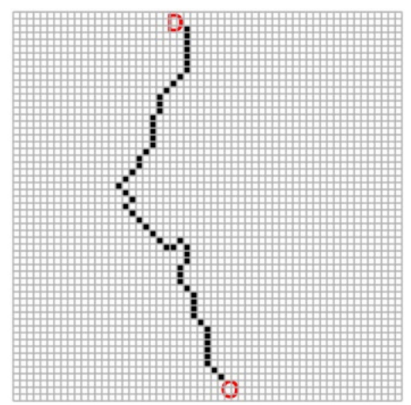

(b)

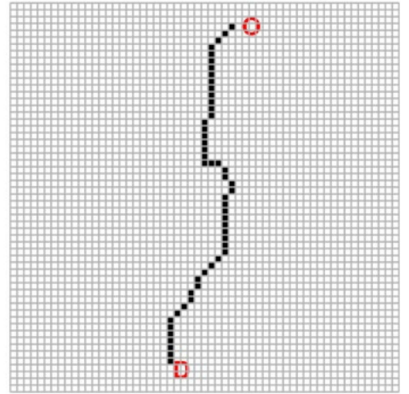

$(\mathbf{f})$

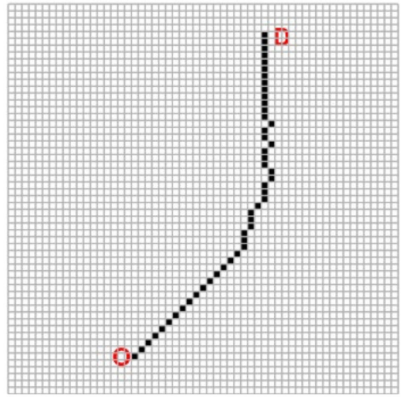

(i)

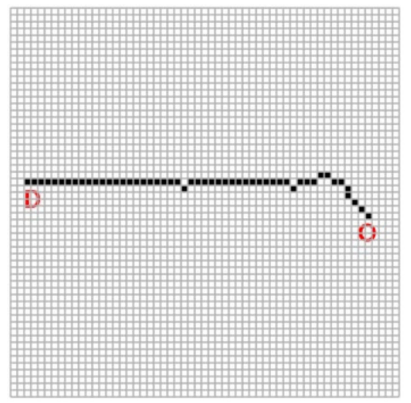

(c)

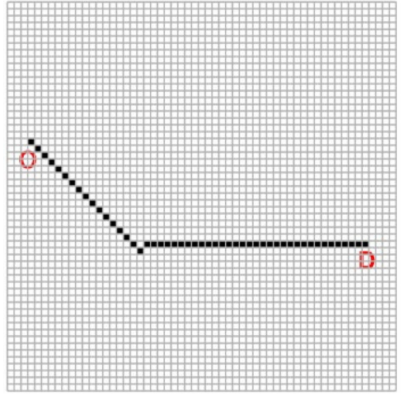

$(\mathrm{g})$

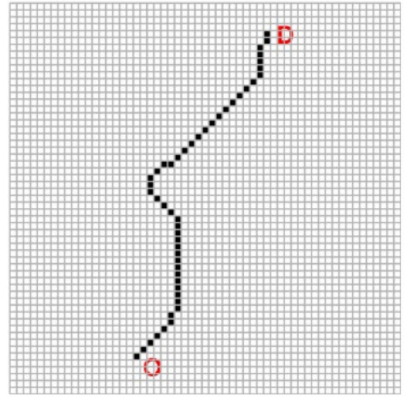

(j)

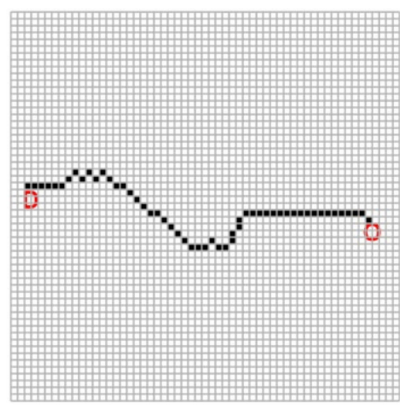

(d)

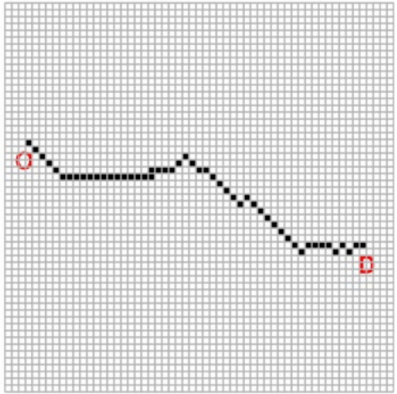

(h)

Figure 19. Comparison of trajectory diagrams of the same pedestrian with or without AFF model (homogeneity). (a) The 1st person has no prediction field; (b) The 1st person has a prediction field; (c) The 15th person has no prediction field; (d) The 15th person has a prediction field; (e) The 30th person has no prediction field; (f) The 30th person has a prediction field; (g) The 45th person has no prediction field; (h) The 45th person has a prediction field; (i) The 60th person has no prediction field; (j) The 60th person has a prediction field.

In addition, the average speed and the change in the distance from the center are selected for comparison between the AFF model of the subdivision level $n=1$ (that is, the pedestrian occupies a single cell) and the traditional model. The comparison chart is shown in Figure 21.

From the comparison of speed changes in Figure 21a,b, it can be seen that, regardless of whether pedestrians are heterogeneous, the average speed change in pedestrians with AFF is compared with the traditional field potential energy model, showing obvious double peaks. The change reflects the process of the generation and dissipation of congestion, while the average speed of pedestrians without a prediction field shows a generally monotonous decline, which cannot reflect the process of generation and dissipation of pedestrian congestion in this scene. Regarding the change in distance from the center (Figure 21c,d), regardless of the presence or absence of the predicted field AFF and the presence or absence of pedestrian heterogeneity, the changes are all U-shaped. Before the minimum value, the average distance of pedestrians from the center showed similar changes, and after the minimum value, regardless of whether the pedestrians are heterogeneous, the average rate of pedestrians leaving the center without the prediction field AFF was significantly greater than that of the prediction field AFF. This shows that pedestrians will still deal with 
possible small-scale congestion after the congestion processing is completed due to the addition of the prediction field, so as to bypass it and reach the end at a speed lower than the average speed of the non-prediction field.

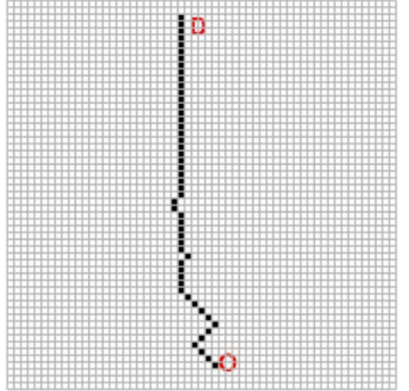

(a)

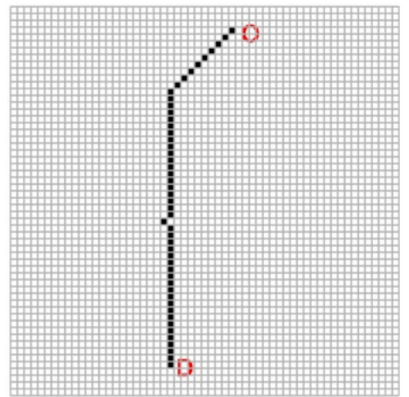

(e)

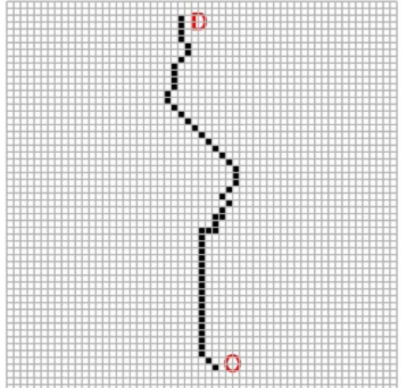

(b)

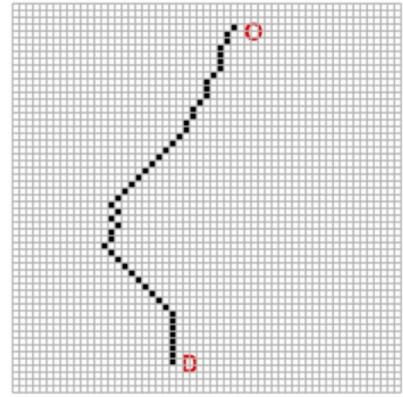

(f)

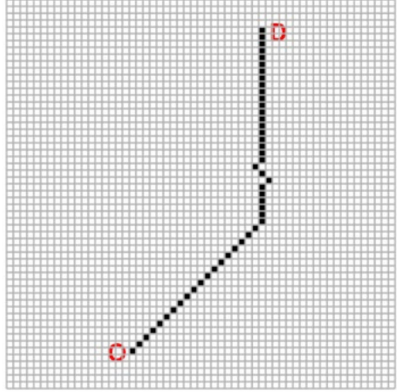

(i)

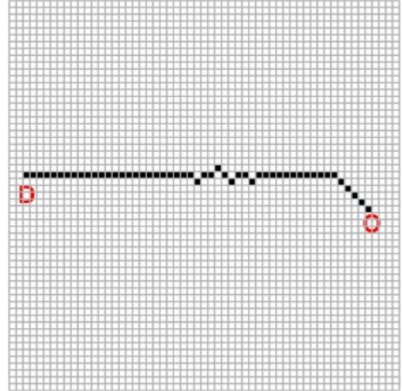

(c)

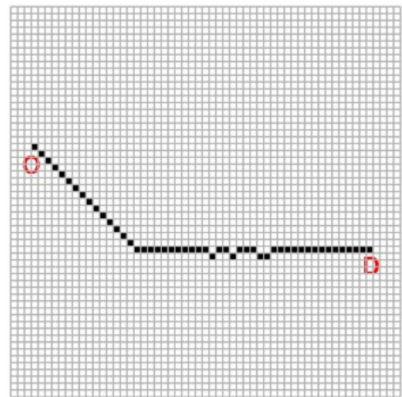

$(\mathrm{g})$

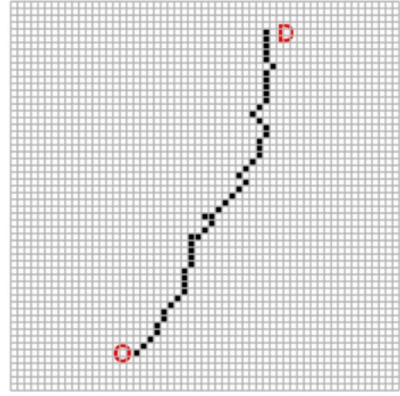

(j)

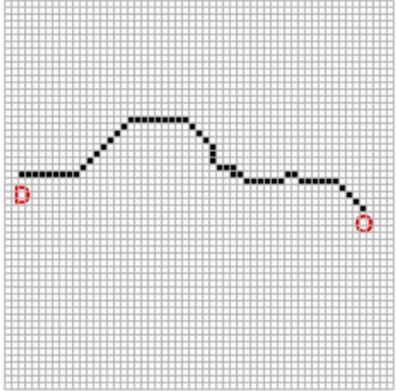

(d)

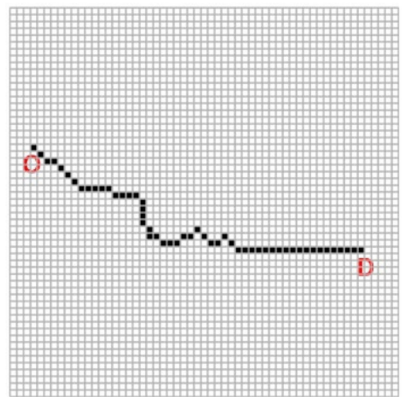

(h)

Figure 20. Comparison of trajectory diagrams of the same pedestrian with or without AFF model (heterogeneity). (a) The 1st person has no prediction field; (b) The 1st person has a prediction field; (c) The 15th person has no prediction field; (d) The 15th person has a prediction field; (e) The 30th person has no prediction field; (f) The 30th person has a prediction field; (g) The 45th person has no prediction field; (h) The 45th person has a prediction field; (i) The 60th person has no prediction field; (j) The 60th person has a prediction field. 


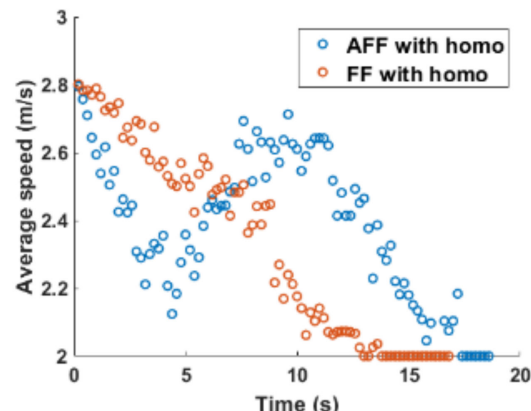

(a)

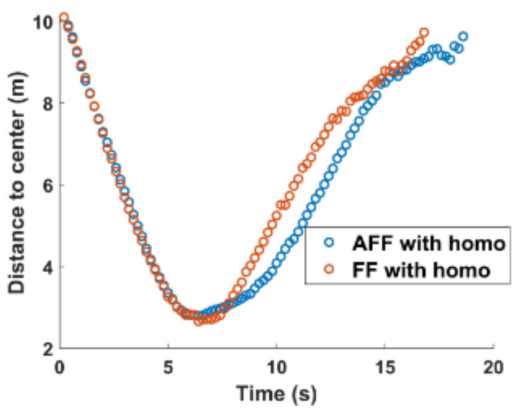

(c)

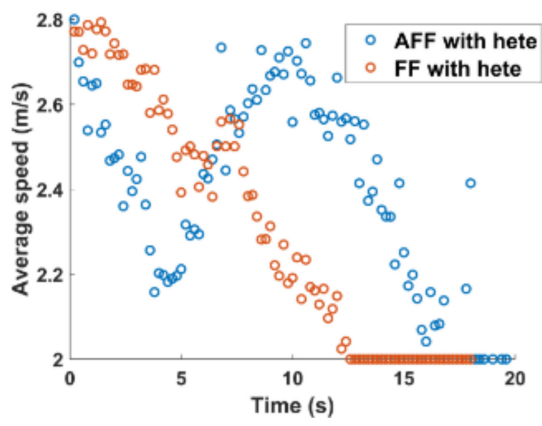

(b)

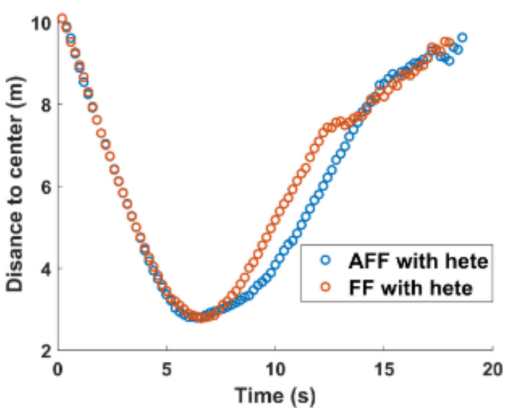

(d)

Figure 21. The influence graph of the presence or absence of AFF on the speed change and the distance change from the center (FF means no predicted field potential energy) (a) Average speed homogeneity; (b) Average speed heterogeneity; (c) Homogeneity of distance from center; (d) Distance heterogeneity.

\section{Discrete Model Comparison}

In this study, the predicted field is integrated into the subdivided field potential energy cellular automata model, and at the same time, considering the pedestrian speed and the heterogeneity of the prediction range, the circumvention behavior of the pedestrian in the circular symmetrical walking experiment is simulated. Compared with the traditional fieldpotential energy cellular automata model that considers pedestrian speed heterogeneity, this improved model can describe the pedestrian's detour behavior more realistically. For this reason, this research also proposes corresponding indicators to compare and analyze the traditional field potential energy cellular automata model and the improved subdivision field potential energy cellular automata model, and compare the advantages and disadvantages of the two types of discrete models in each indicator.

Since the specific behavior of pedestrians is the most intuitive reflection of their trajectory, we select pedestrian trajectory characteristics as the main indicators to describe pedestrian detour behavior, including the spatial dimension indicators of pedestrian trajectory such as travel time distribution, path length distribution, etc., time dimension Indicators such as density time series indicators, average speed time series indicators, and time series indicators of the average distance from the center, etc.

Since the cellular automata model is discrete in time, and as the degree of subdivision increases, the time step of the simulation will also become smaller, and the time sequence requires the two sequences to be at the same time step. At the same time, since the purpose of the simulation is to be consistent with the actual pedestrian trajectory characteristics, in order to compare the gap between the simulated and experimental trajectory characteristics, we select the subdivision $n=5$, and its corresponding time step $\Delta t=0.04 \mathrm{~s}$, which is consistent with the experiment. The data are consistent (experimental data video data are $25 \mathrm{fps}$ ). Similarly, whether there is a heterogeneous subdivision cellular automata model is used as a comparative evaluation of the simulation of this experiment. We apply Gaussian 
fitting to the time series of pedestrian average speeds with different subdivisions, as shown in Figure 22.

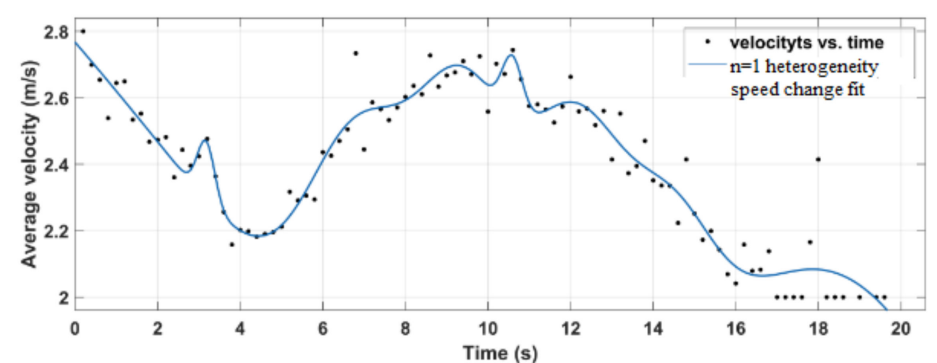

(a)

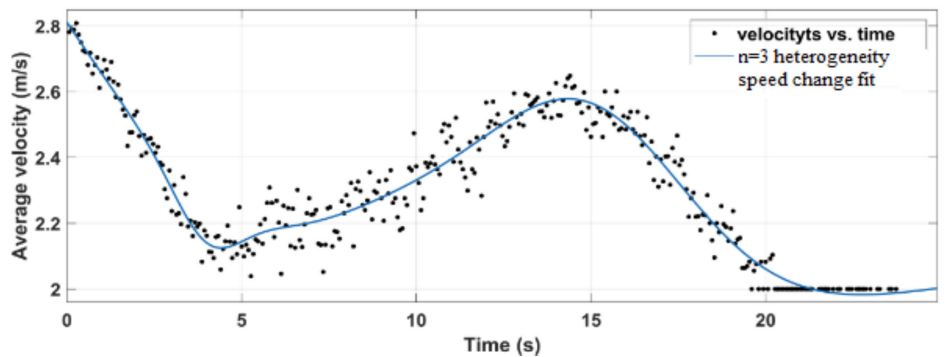

(b)

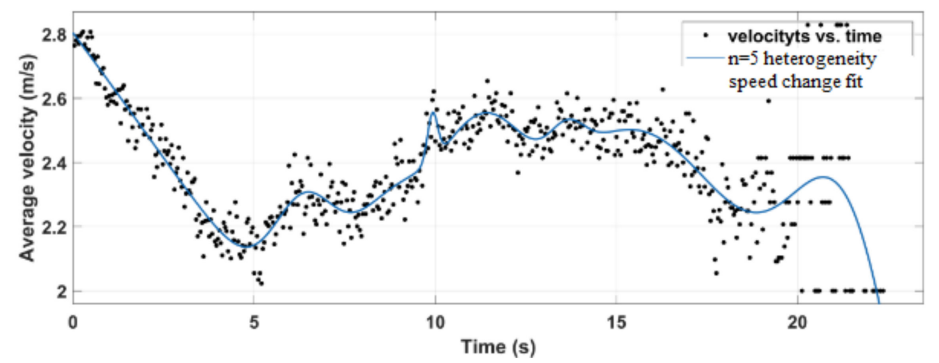

(c)

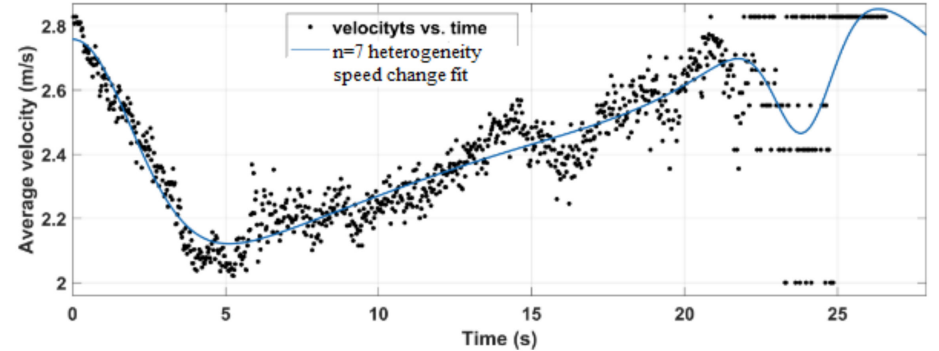

(d)

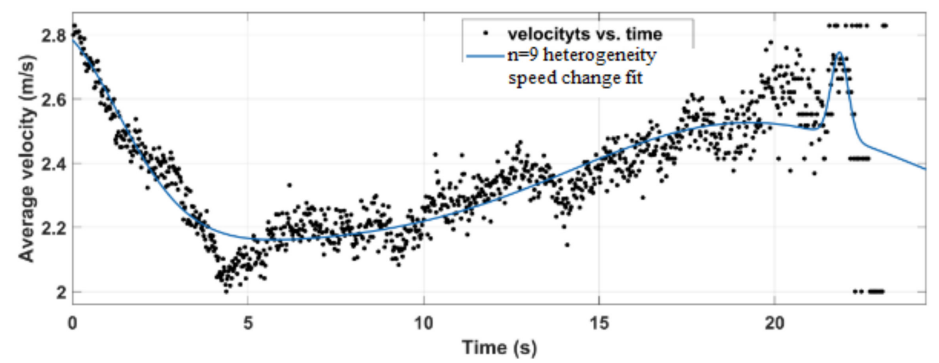

(e)

Figure 22. Cont. 


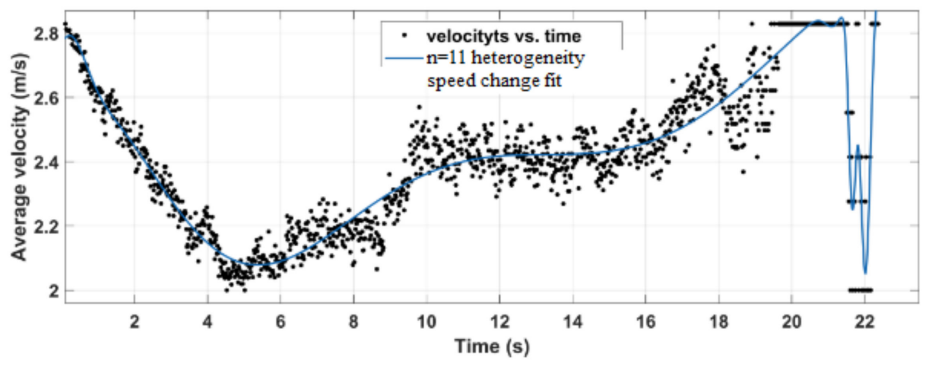

$(\mathbf{f})$

Figure 22. Gaussian fitting diagram of the speed change in the heterogeneous pedestrian AFF model under each subdivision. (a) when subdivision $\mathrm{n}=1$; (b) when subdivision $\mathrm{n}=3$; (c) when subdivision $\mathrm{n}=5$; (d) when subdivision $\mathrm{n}=7$; $(\mathbf{e})$ when subdivision $\mathrm{n}=9$; $(\mathbf{f})$ when subdivision $\mathrm{n}=11$.

At the same time, the Gaussian fitting parameters of the heterogeneous pedestrian speed changes under each subdivision are shown in Table 2.

Table 2. The sum of square error SSE, multiple measurement coefficient R-square, and root mean square error RMSE table of heterogeneous pedestrian speed change fitting under each subdivision.

\begin{tabular}{cccc}
\hline Subdivision & SSE & R-Square & RMSE \\
\hline $\mathrm{n}=1$ & 0.3638 & 0.9273 & 0.0711 \\
$\mathrm{n}=3$ & 0.8995 & 0.9394 & 0.0524 \\
$\mathrm{n}=5$ & 4.8461 & 0.7109 & 0.0966 \\
$\mathrm{n}=7$ & 9.2808 & 0.7908 & 0.1021 \\
$\mathrm{n}=9$ & 8.9996 & 0.7525 & 0.0938 \\
$\mathrm{n}=11$ & 6.6615 & 0.8916 & 0.0745 \\
\hline
\end{tabular}

When the subdivision $n=5$, taking the heterogeneous pedestrian as an example, the Gaussian fitting function of the simulated average speed time series is:

$$
\begin{aligned}
f(x)=a 1 * \exp & \left(-\left(\frac{x-b 1}{c 1}\right)^{2}\right)+a 2 * \exp (-((x-b 2) / c 2) 2)+a 3 * \exp (-((x-b 3) / c 3) 2)+a 4 \\
& * \exp (-((x-b 4) / c 4) 2)+a 5 * \exp \left(-((x-b 5) / c 5)^{2}\right)+a 6 * \exp (-((x \\
& -b 6) / c 6)^{2}+a 7 * \exp \left(-((x-b 7) / c 7)^{2}\right)
\end{aligned}
$$

The parameter values are:

$$
\begin{aligned}
a 1=2.312 ; b 1 & =19.12 ; c 1=6.325 * 10^{6} ; a 2=0 ; b 2=32.41 ; c 2=2.22 * 10^{-14} ; a 3=0.5142 ; b 3 \\
& =30.88 ; c 3=8.778 ; a 4=0 ; b 4=30.18 ; c 4=2.22 * 10^{-14} ; a 5=0.3623 ; b 5 \\
& =20.17 ; c 5=5.698 ; a 6=-0.3513 ; b 6=29.16 ; c 6=0.01995 ; a 7=0.1603 ; b 7 \\
& =13.16 ; c 7=2.536 ;
\end{aligned}
$$

The selected experiment is the third experiment of $64 \mathrm{p}-10 \mathrm{~m}$, and the Gaussian fitting of the average speed of pedestrians is shown in Figure 23.

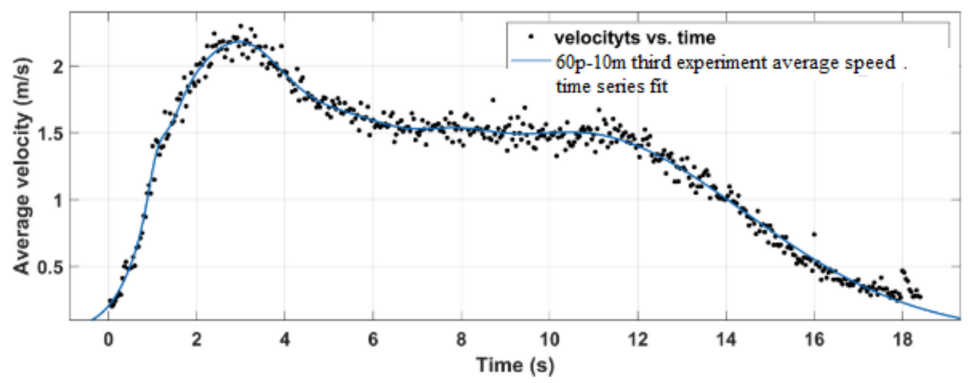

Figure 23. Schematic diagram of Gaussian fitting of experimental pedestrian speed changes.

Correspondingly, the Gaussian fitting function of the average speed of pedestrians is: 


$$
\begin{aligned}
& f(x)=a 1 * \exp \quad(-((x-b 1) / c 1) 2)+a 2 * \exp (-((x-b 2) / c 2) 2)+a 3 * \exp (-((x-b 3) / c 3) 2) \\
& +a 4 * \exp (-((x-b 4) / c 4) 2)+a 5 * \exp (-((x-b 5) / c 5) 2)+a 6 * \exp (-((x \\
& -b 6 / c 6) 2 \text { ) } \\
& \text { The parameter values are: } \\
& a 1=0.4263 ; b 1=1.475 ; c 1=0.8911 ; a 2=1.759 ; b 2=2.729 ; c 2=1.72 ; a 3=0.2086 ; b 3 \\
& =1.096 ; c 3=0.2614 ; a 4=0.2017 ; b 4=7.868 ; c 4=1.255 ; a 5=0.8865 ; b 5 \\
& =5.305 ; c 5=1.972 ; a 6=1.501 ; b 6=10.57 ; c 6=5.435 \text {; }
\end{aligned}
$$

The time series of the average distance from the center of the selected $64 \mathrm{p}-10 \mathrm{~m}$ third experiment is shown in Figure 24.

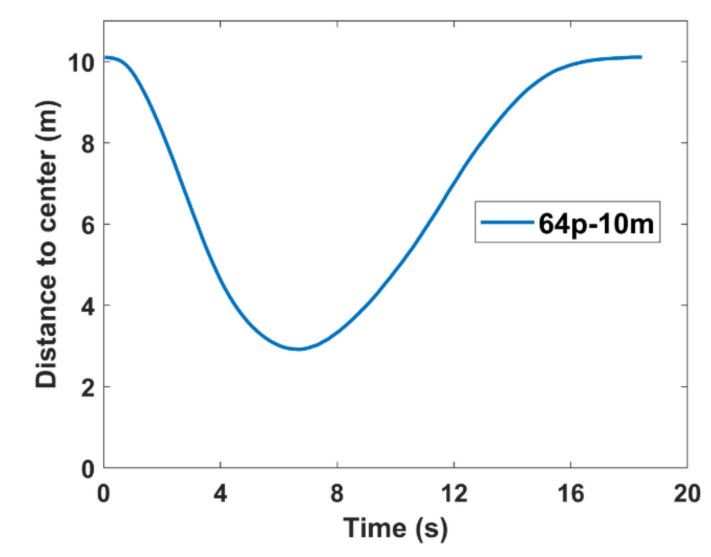

Figure 24. Time series diagram of the distance between the experimental pedestrian and the center.

The selected indicators are path length, travel time distribution index and average speed, and distance from the center time series index. The distribution index reflects the characteristics of pedestrian trajectory in space, and the time series indicator reflects the characteristics of pedestrian trajectory in time. Using the distribution index evaluation method based on the Kolmogorov-Smirnov test and the time series evaluation method based on the dynamic time warping algorithm proposed by Xiao et al. [16], we obtain the similarity score between the simulation data and the experimental data with or without pedestrian heterogeneity. The score radar chart of the indicator is shown in Figure 25.

It can be seen from the score radar chart that the subdivided cellular automata model has very low similarity to the experiment in trajectory characteristics, and the score on the time series index is slightly higher than the distribution index, combined with Section 3.2 to determine whether there is heterogeneity. The cumulative probability density map (see Figures 11 and 12) and the analysis of the trajectory map show that, with the addition of the prediction field, pedestrians still go straight to the center, and then show a unified detour pattern to bypass the central area and move toward the target cell, making it cumulative. The probability density presents a ring shape near the center, which shows that the pedestrian walking rules based on field potential energy cannot effectively reflect the macroscopic decision of pedestrians facing congestion. Correspondingly, the pedestrian heterogeneity has little effect on the simulation score, and the scores of various indicators are only slightly higher than the homogeneous pedestrian simulation score. 


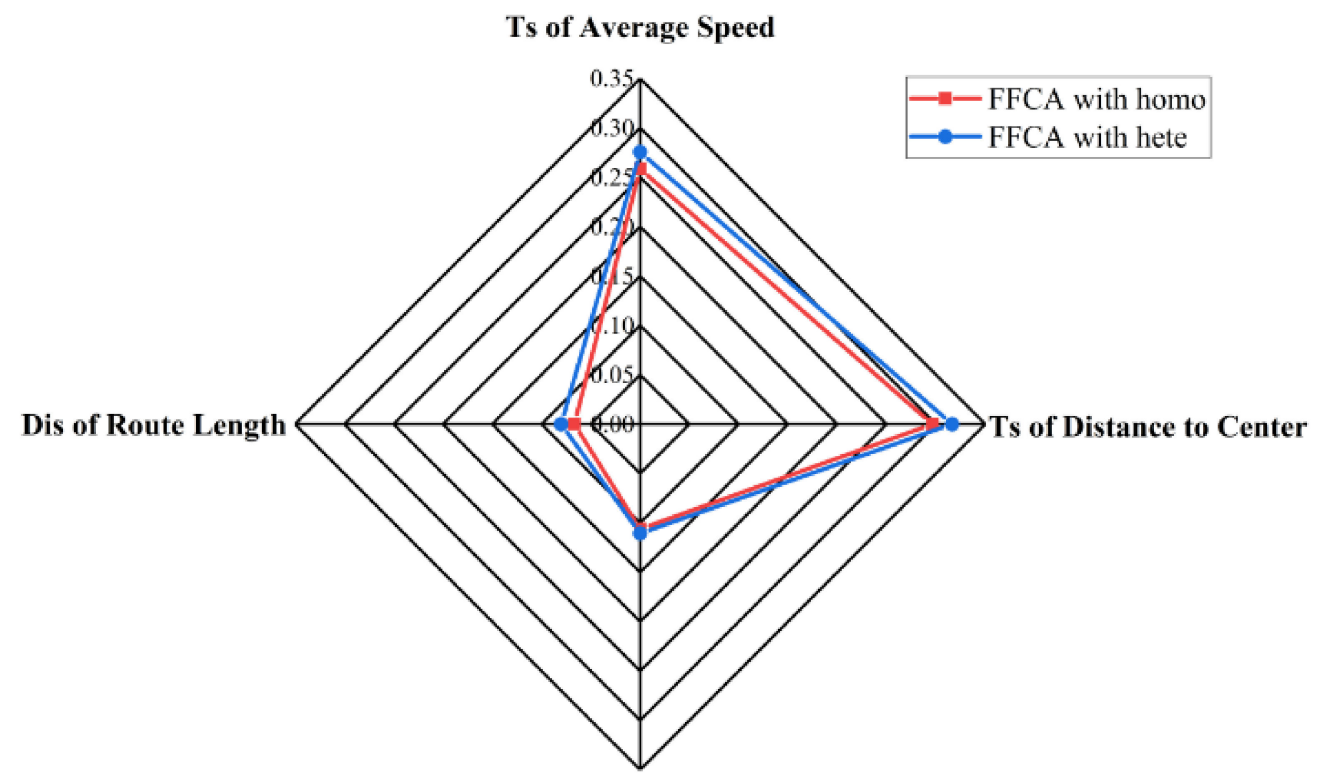

Dis of Travel Time

Figure 25. Radar chart of simulation score of cellular automata model with or without pedestrian heterogeneity segmentation. (FFCA means floor field cellular automata model, Ts means time series, Dis means distribution characteristics).

\section{Conclusions}

In this study, we used subdivided cells to accurately describe the distribution of pedestrian positions, update the position of pedestrians simultaneously, and reduce the timescale of pedestrian movement of a single cell and the probability of pedestrian movement to achieve pedestrian velocity heterogeneity and improve the prediction field. In order to realize the heterogeneity of the pedestrian prediction range, a synchronously updated subdivision cell prediction field automata model is obtained to realize the description of the pedestrian detour behavior in the circular symmetry experiment.

The heterogeneity of pedestrians includes the heterogeneity of prediction range and speed. The prediction range is related to the expected speed. The greater the expected speed of pedestrians, the larger the prediction range, and vice versa. There is no heterogeneity and the influence of the predicted field on the simulation. Through the analysis of the cumulative density, the average speed change, the average distance change from the center, and the related trajectory, the analysis shows that the presence or absence of subdivision has a significant impact on the cumulative density of the simulation. The cumulative density of unsubdivided cells presents a ring near the center, while the subdivision makes the homogeneous pedestrian ring disappear and clusters in the center, while the heterogeneous pedestrian ring becomes smaller. Pedestrian heterogeneity has a greater impact on simulation. The main manifestation is that the heterogeneous cumulative density presents a near-center circular distribution. This shows that, due to the heterogeneity of the pedestrian prediction range, some people first predict the occurrence of congestion, meaning it is reduced. Other directions to detour will then be slowly chosen, and then other pedestrians who arrive one after another will also predict the occurrence of congestion near the center area, thus presenting a unified detour pattern. Finally, compared to the simulation without the prediction field, the cumulative density forms a ring near the center regardless of pedestrian heterogeneity, and the existence of the prediction field makes the trajectory of pedestrians have a greater tendency to detour.

Finally, an index for evaluating the temporal and spatial characteristics of pedestrian trajectories is proposed. The spatial characteristics include travel time distribution and path length distribution, while time characteristics include distance from the center time series and average speed time series. The experimental trajectory data and simulated trajectory 
data are distributed through the KS test. As a similarity test, the time series feature similarity test is carried out through the DTW method, and the comprehensive evaluation of whether there is heterogeneity in the subdivision prediction field cellular automata model simulation is good or bad in the trajectory characteristics. The evaluation results show that, compared with homogeneous pedestrians, heterogeneous pedestrians make the characteristics of the simulated trajectory more similar to the experimental trajectory.

In the circle antipode experiment, pedestrians are almost faced with a completely symmetrical situation, and pedestrians have different detour patterns in trajectory characteristics, which are heterogeneous. This study explored the influence of pedestrian speed heterogeneity on pedestrian circumvention behavior in a circular walking scene by subdividing the cell size prediction field potential energy model. The research results show that pedestrian heterogeneity makes pedestrian trajectory characteristics and experiments the similarity score is better, which is more in line with the characteristics of the experimental pedestrian trajectory. Of course, this study did not compare the pros and cons of this model and the traditional field potential energy model in terms of trajectory time and space indicators, and at the same time, there are fewer evaluation indicators, which fails to fully display the characteristics of pedestrian trajectories. In future studies, other characteristics of pedestrian trajectories will be considered, as well as a comparison of different models.

Author Contributions: Conceptualization, M.L.; methodology, M.L.; software, P.S.; validation, J.L., M.L. and P.S.; formal analysis, J.L. and P.S.; data curation, J.L., P.S. and M.L.; writing-original draft preparation, J.L. and P.S.; writing-review and editing, J.L. and M.L. All authors have read and agreed to the published version of the manuscript.

Funding: This research received no external funding.

Institutional Review Board Statement: Not applicable.

Informed Consent Statement: Not applicable.

Data Availability Statement: Not report any data.

Acknowledgments: The authors send their appreciation to the Central South University.

Conflicts of Interest: The authors declare no conflict of interest.

\section{References}

1. Henderson, L.F. The statistics of crowd fluids. Nature 1971, 229, 381-383. [CrossRef] [PubMed]

2. Burstedde, C.; Kirchner, A.; Klauck, K.; Schadschneider, A.; Zittartz, J. Cellular Automaton Approach to Pedestrian DynamicsApplications. Pedestr. Evacuation Dyn. 2002, 15, 87-97.

3. Chen, M.; Han, D.; Zhang, H. Research on a multi-grid model for passenger evacuation in ships. J. Mar. Sci. Appl. 2011, 10, 340-346. [CrossRef]

4. Kirchner, A.; Klupfel, H.; Nishinari, K.; Schadschneider, A.; Schreckenberg, M. Discretization effects and the influence of walking speed in cellular automata models for pedestrian dynamics. J. Stat. Mech.-Theory Exp. 2004, 2004, P10011. [CrossRef]

5. Suma, Y.; Yanagisawa, D.; Nishinari, K. Anticipation effect in pedestrian dynamics: Modeling and experiments. Phys. A Stat. Mech. Appl. 2012, 391, 248-263. [CrossRef]

6. Guo, R.Y.; Huang, H.-J. A mobile lattice gas model for simulating pedestrian evacuation. Physica A 2008, 387, 580-586. [CrossRef]

7. Chen, L.; Tang, T.-Q.; Huang, H.-J.; Wu, J.-J.; Song, Z. Modeling pedestrian flow accounting for collision avoidance during evacuation. Simul. Model. Pract. Theory 2018, 82, 1-11. [CrossRef]

8. Zhou, X.; Hu, J.; Ji, X.; Xiao, X. Cellular automaton simulation of pedestrian flow considering vision and multi-velocity. Physica A 2015, 14, 982-992. [CrossRef]

9. Davidich, M.; Geiss, F.; Mayer, H.G.; Pfaffinger, A.; Royer, C. Waiting zones for realistic modelling of pedestrian dynamics: A case study using two major German railway stations as examples. Transp. Res. Part C-Emerg. Technol. 2013, 37, 210-222. [CrossRef]

10. Fu, Z.; Deng, Q.; Schadschneider, A.; Li, Y.; Luo, L. Resolution of deadlocks in a fine discrete floor field cellular automata model-modeling of turning and lateral movement at bottlenecks. J. Stat. Mech. Theory Exp. 2019, 2019, 123402. [CrossRef]

11. Zhuang, Y.; Liu, Z.; Schadschneider, A.; Yang, L.; Huang, J. Exploring the behavior of self-organized queuing for pedestrian flow through a non-service bottleneck. Phys. A Stat. Mech. Appl. 2021, 562, 12518. [CrossRef]

12. Guo, R.-Y. New insights into discretization effects in cellular automata models for pedestrian evacuation. Physica A 2014, 400, 1-11. [CrossRef] 
13. Fu, Z.; Jia, Q.; Chen, J.; Ma, J.; Han, K.; Luo, L. A fine discrete field cellular automaton for pedestrian dynamics integrating pedestrian heterogeneity, anisotropy, and time-dependent characteristics. Transp. Res. Part C Emerg. Technol. 2018, 91, 37-61. [CrossRef]

14. Li, C.-Y.; Yang, R.-Y.; Chen, L.; Tang, T.-Q. A boarding model for heterogeneous passengers on the platform of high-speed railway station. Simul. Model. Pract. Theory 2021, 106, 102188. [CrossRef]

15. Guo, N.; Hao, Q.-Y.; Jiang, R.; Hu, M.-B.; Jia, B. Uni- and bi-directional pedestrian flow in the view-limited condition: Experiments and modeling. Transp. Res. Part C-Emerg. Technol. 2016, 71, 63-85. [CrossRef]

16. Xiao, Y.; Gao, Z.; Jiang, R.; Li, X.; Qu, Y.; Huang, Q. Investigation of pedestrian dynamics in circle antipode experiments: Analysis and model evaluation with macroscopic indexes. Transp. Res. Part C-Emerg. Technol. 2019, 103, 174-193. [CrossRef] 